\title{
Robust adaptive algorithms for underwater acoustic channel estimation and their performance analysis
}

\author{
Dariush Kari $^{\mathrm{a}, *}$, Iman Marivani ${ }^{\mathrm{a}}$, Farhan Khan ${ }^{\mathrm{a}}$, Muhammed Omer Sayin ${ }^{\mathrm{b}}$, \\ Suleyman Serdar Kozat ${ }^{a}$ \\ a Department of Electrical and Electronics Engineering, Bilkent University, Ankara, 06800, Turkey \\ b Coordinated Science Laboratory, University of Illinois at Urbana-Champaign, Urbana, IL 61801 USA
}

\section{A R T I C L E I N F O}

\section{Article history:}

Available online 24 May 2017

\section{Keywords:}

Underwater communications

Robust channel estimation

Logarithmic cost function

Impulsive noise

Performance analysis

Carrier offsets

\begin{abstract}
A B S T R A C T
We introduce a novel family of adaptive robust channel estimators for highly challenging underwater acoustic (UWA) channels. Since the underwater environment is highly non-stationary and subjected to impulsive noise, we use adaptive filtering techniques based on minimization of a logarithmic cost function, which results in a better trade-off between the convergence rate and the steady state performance of the algorithm. To improve the convergence performance of the conventional first and second order linear estimation methods while mitigating the stability issues related to impulsive noise, we intrinsically combine different norms of the error in the cost function using a logarithmic term. Hence, we achieve a comparable convergence rate to the faster algorithms, while significantly enhancing the stability against impulsive noise in such an adverse communication medium. Furthermore, we provide a thorough analysis for the tracking and steady-state performances of our proposed methods in the presence of impulsive noise. In our analysis, we not only consider the impulsive noise, but also take into account the frequency and phase offsets commonly experienced in real life experiments. We demonstrate the performance of our algorithms through highly realistic experiments performed on accurately simulated underwater acoustic channels.
\end{abstract}

(c) 2017 Elsevier Inc. All rights reserved.

\section{Introduction}

Underwater acoustic (UWA) communication has attracted much attention in recent years due to proliferation of new and exciting applications such as marine environmental monitoring and sea bottom resource exploitation [1-3]. However, due to the constant movement of waves, multi-path propagation, large delay spreads, Doppler effects, and frequency dependent propagation loss [3,4], the underwater acoustic channel is considered as one of the most detrimental communication mediums in use today [4,5]. In these mediums, channel equalization [6,7] plays a key role in providing reliable high data rate communication [3]. Note that, in order to combat the effects of long and time varying channel impulse response (CIR), orthogonal frequency division multiplexing (OFDM) seems to be an elegant solution [8]. However, one needs an accurate estimate of the time varying channel to be used for OFDM as well as equalization. Furthermore, due to rapidly changing and

\footnotetext{
* Corresponding author.

E-mail addresses: kari@ee.bilkent.edu.tr (D. Kari), marivani@ee.bilkent.edu.tr (I. Marivani), khan@ee.bilkent.edu.tr (F. Khan), sayin2@illinois.edu (M.O. Sayin), kozat@ee.bilkent.edu.tr (S.S. Kozat).
}

unpredictable nature of underwater environment, such processing should be adaptive [3]. Nevertheless, the impulsive nature of the ambient noise in UWA channels introduces stability issues for adaptive channel estimation $[9,10]$. To this end, we propose and analyze the performance of a family of new adaptive linear channel estimators, which provides a relatively fast convergence rate as well as strong stability against the ambient noise in the UWA channels.

Although the additive white Gaussian noise (AWGN) model is widely used in digital and wireless communication contexts, this model is insufficient to appropriately address the ambient noise in UWA channels [11-13]. For example, in warm shallow waters, the high frequency noise component is dominated by the "impulsive noise" [14-17] resulted from numerous noise sources such as marine life, shipping traffic, underwater explosives, and offshore oil exploration-production [18]. The impulsive noise consists of relatively short duration, infrequent, high amplitude noise pulses. In this paper, in order to rectify the undesirable effects of UWA channels, especially to mitigate the effects of the impulsive noise, we introduce a radical approach to adaptive channel estimation.

In [19], the authors propose a low-complexity decision feedback equalizer, which employs a sphere detection-Viterbi algorithm 
(SDVA) with two radii in a decision feedback equalization (DFE). [19] provides a simple operating condition for the SDVA, under which the algorithm only searches a small fraction of the lattice points lying between two radii, hence, provides a lower complexity. However, the impulsive noise in underwater channels degenerates the performance of the SDVA-algorithm. Thus, some preprocessing steps can reduce the impulsive noise effect and improve the performance. Also, in [20], for localization of multiple acoustic sources in an impulsive-noise environment, the authors employ a data-adaptive zero-memory nonlinear preprocessor to process each sample of the contaminated data to obtain less-noisy data. Specifically, if the sample's value is less than a threshold, they assume it is not affected by the impulsive noise, hence, its value does not change during the process. However, samples above the threshold are assumed to be corrupted by a large noise impulse and are suppressed. Note that the threshold is determined based on the noise statistics, hence, one has to estimate the noise statistics.

Linear adaptive channel estimation methods (e.g., sign algorithm (SA), least mean squares (LMS) or least mean fourth (LMF) algorithms [21,22]) are the simplest as well as low complexity methods. However, the conventional linear estimators either have a slow convergence speed (e.g., sign algorithm (SA)) or suffer from stability issues (e.g., LMF) in impulsive noise environments, hence, cannot fully address the problem [23]. These methods are commonly based on minimization of a cost function of the form $C\left(e_{m}\right) \triangleq E\left[\left|e_{m}\right|^{k}\right]$ (where $E[$.] indicates the expectation), using the stochastic gradient descent method $[24,25]$. However, there is always a trade-off between the "robustness" of such algorithms against impulsive noise and their convergence speed [23]. In this sense, the algorithms that use lower powers of the error as the cost function (e.g., SA [25]) provide a better robustness, while, the algorithms based on higher powers of the error, usually exhibit faster convergence [23].

The mixed norm algorithms, combining different norms of the error in the cost function, are used to achieve a better trade-off between robustness and convergence speed [26,27]. Nevertheless, optimization of the combination parameters in such algorithms needs "a priori" knowledge of the noise and input signal statistics [23]. On the contrary, the mixture of experts algorithms [28-30], adaptively learn the best combination parameters. However, such algorithms are infeasible in UWA scenarios due to the high computational complexity resulted from running several different algorithms in parallel [23,31].

Recently, in [32] and [33], the authors proposed recursive least squares (RLS)-type robust adaptive estimation and equalization methods, which leverage the sparsity of the underwater channels by adding an $l_{0}$-norm to the cost function. However, the methods in [32] and [33] are not completely adaptive in the sense that they need a few threshold values to be determined in advance, and the values of these thresholds are highly dependent on the transmitted data statistics. In [34], a hyperbolic function, e.g., $C\left(e_{m}\right)=\cosh \left(e_{m}\right)$, is used as the cost function to inherently combine different powers of the error. Moreover, [35] uses a sparse least mean $p$-power (LMP) algorithm to adaptively provide a robust estimate of the underwater channel.

In this paper, in order to obtain a comparable performance to the robust algorithms, while retaining the fast convergence of conventional least square methods, we use a logarithmic function as a regularization term in the cost function of the well-known adaptive methods. In this sense, we choose a conventional method that uses a power of the error as the cost, e.g, least mean squares (LMS), and improve that method through adding a logarithmic term to its cost function. Due to the characteristics of the logarithmic functions, when the error is high, e.g., when there is an impulse, the cost function resembles the cost function of the original method, while for the small errors a correction term is added.
The correction term includes the higher powers of the error, which yields a faster convergence. Hence, we intrinsically mitigate the effect of the impulsive noise pulses and provide an improved robustness, while increase the convergence speed when there is no impulsive noise.

Specifically, we present first and second order channel estimation algorithms using the logarithmic cost. The first order methods, logarithmic cost least mean absolute (LCLMA) and logarithmic cost least mean squares (LCLMS), are based on the first and second powers of the error as in SA and LMS. Similarly, we use the first and second powers of the error to introduce logarithmic cost recursive least squares (LCRLS), and logarithmic cost recursive least absolutes (LCRLA), which are second order (RLS-type) algorithms. An alternative design could use a conventional equalizer, aided by a preprocessing step that performs impulsive noise reduction. For instance, if OFDM is used, the probability density function of the noise-free signal is approximately Gaussian, since an OFDM signal consists of a sum of many independent and identically distributed signals and due to the Central Limit Theorem, results in a nearly Gaussian distributed signal [36]. Therefore, a Bayesian minimum mean squared error (MMSE) approach, applied to the signal-plusimpulsive-noise, would reduce the impulsive noise $[37,38]$. Note that this approach would maximize the SNR at the output of the preprocessing step [38]. After reducing the impulsive noise in the preprocessing step, a conventional equalizer (designed for Gaussian noise) could be employed.

Moreover, we provide a thorough analysis of the tracking and steady state performance of our algorithms. In these analyses, in order to be consistent with the real world UWA channels, we assume that there are frequency and phase offsets as well as impulsive noise in the channel. For the first order algorithms, we avoid mentioning all of the intermediate steps of the analyses, instead we discuss the important steps, use the results of [39], and establish the final results based on them. However, for the second order analyses, we completely explain the steps since it is a nontrivial extension of the existing analysis methods. We show the improved performance of our algorithms and the correctness of our analyses through highly realistic simulations.

The paper is organized as follows: In Section 2, we introduce the notation and describe the problem mathematically. Then, in Section 3, we provide a family of stable and fast converging channel estimators based on logarithmic cost functions. We provide the performance analysis for our methods in Section 4. We demonstrate the performance of the presented methods through highly realistic simulations in Section 5 and conclude the paper with several remarks in Section 6 .

\section{Problem description}

\subsection{Notations}

All vectors are column vectors and are denoted by boldface lower case letters and all matrices are denoted by boldface upper case letters. For a vector $\boldsymbol{x}, \boldsymbol{x}^{H}$ is the Hermitian transpose. We denote the all zero vector of size $L \times 1$ by $\mathbf{0}_{L}$. In addition, $E[x]$ denotes the expectation of the random variable $x$, and $\operatorname{Tr}(\boldsymbol{A})$ denotes the trace of the matrix $\boldsymbol{A}$. Also, the discrete time variables are shown as subscripts, while the continuous time variable $t$ is parenthesized. Furthermore, for a vector $\boldsymbol{w}$ the squared $l_{2}$-norm is defined as $\|\boldsymbol{w}\|^{2} \triangleq \boldsymbol{w}^{H} \boldsymbol{w}$, and the weighted squared $l_{2}$-norm is defined as $\|\boldsymbol{w}\|_{\boldsymbol{P}}^{2} \triangleq \boldsymbol{w}^{T} \boldsymbol{P} \boldsymbol{w}$, where $\boldsymbol{P}$ is a positive definite weighting matrix. 


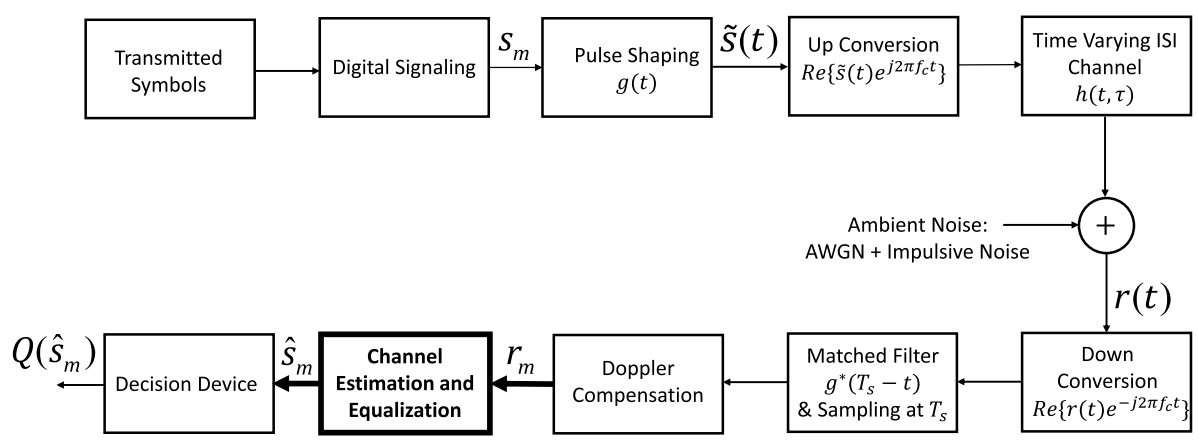

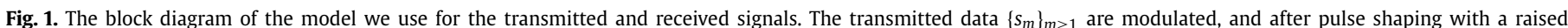

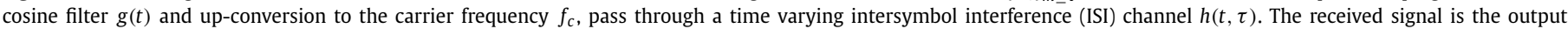

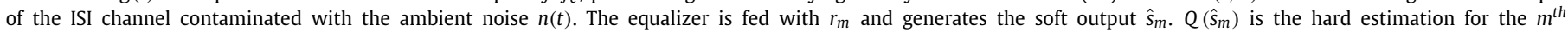
transmitted symbol. We use the hard estimates $Q\left(\hat{s}_{m}\right)$ for adapting the channel estimator, and use the channel estimation to reduce the ISI.

\subsection{Setup}

As depicted in Fig. 1, we denote the received signal by $r(t)$, $r(t) \in \mathbb{R}$, and our aim is to determine the transmitted symbols $\left\{s_{m}\right\}_{m \geq 1}$. To transmit the symbols $\left\{s_{m}\right\}_{m \geq 1}$, we use the raised cosine pulse shaping filter $g(t)$, which generates the signal $\tilde{s}(t)$, and then up-convert the signal to the carrier frequency $f_{c}$, and send it through the channel. Using the linear time varying convolution between $\tilde{s}(t)$ and $h(t, \tau)[40]$, the received signal at time $t$ is

$r(t)=\int_{0}^{\tau_{\max }} \tilde{s}(t-\tau) h(t, \tau) d \tau+n(t)$,

where $\tilde{s}(t)$ is the transmitted signal after pulse shaping at time $t$. $h(t, \tau)$ indicates the CIR at time $t$ corresponding to the impulse sent at time $t-\tau$ and $\tau_{\max }$ shows the maximum delay spread of the channel. Also, $n(t)$ is the ambient noise of the channel, which is represented as

$n(t)=n_{g}(t)+n_{i}(t)$,

where $n_{g}(t)$ indicates the white Gaussian part of the noise and $n_{i}(t)$ indicates the impulsive part. Note that the effects of time delay and phase deviations are usually addressed at the front-end of the receiver, hence, in this paper, we do not deal with these problems explicitly. We then sample the received signal every $T_{s}$ seconds ( $T_{S}$ is the symbol duration) such that the discrete time channel model is represented as [40]

$r_{m}=\sum_{k=0}^{L-1} s_{m-k} h_{m, k}+n_{m}$,

and

$n_{m}=n_{g, m}+n_{i, m}$,

where $L=\left\lfloor\tau_{\max } / T_{S}\right\rfloor$ indicates the length of the CIR. In the following sections, we use the discrete time model of the channel to address the estimation and equalization problems. Note that (3) is a causal representation of the channel, i.e., the channel output at time $m$ depends only on the transmitted symbols before $m$.

\subsection{Doppler compensation}

In order to compensate for the Doppler effects, a linear interpolation method is used to convert the sampling rate of the signal [41]. The complex baseband signal (after the matched filter), is sampled at four times the symbol rate and shown by $y_{m^{\prime}}$. The output of the interpolator is then down-sampled to two samples

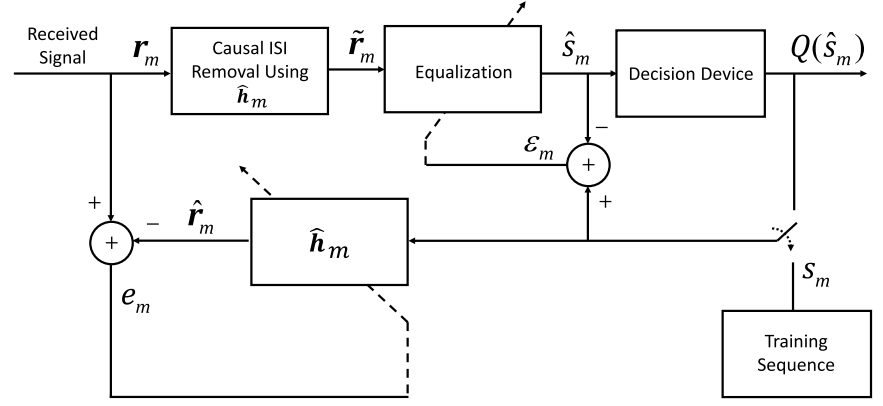

Fig. 2. The block diagram of our adaptive channel estimator and equalizer. We present new algorithms for the channel estimation block (which determines how should $\hat{\boldsymbol{h}}_{m}$ be updated based on the error amount $e_{m}$ ), which yields improved performance over the conventional methods in the impulsive noise environments.

per symbol shown by $r_{m^{\prime \prime}}$, which is finally used as the input to the equalizer [41]. The adaptive resampling algorithm is given as

$$
\begin{aligned}
r_{m^{\prime \prime}} & =\left(I_{m}-1\right) y_{m^{\prime}+1}+I_{m} y_{m^{\prime}}, \\
I_{m+1} & =I_{m}+K_{p} \phi_{m}, \\
\phi_{m} & =\arg \left\{\bar{s}_{m} \hat{s}_{m}^{*}\right\},
\end{aligned}
$$

where $K_{p} \in\left[10^{-6}, 10^{-4}\right]$ is the phase tracking constant, and $\hat{s}_{m}$ is the output of the equalizer. In addition, in the training phase, $\bar{s}_{m}=s_{m}$, and in the decision directed phase, $\bar{s}_{m}$ indicates the hard estimate of the $s_{m}$. Also, note that $m^{\prime} \in\{1,3,5, \ldots\}$ and $m^{\prime \prime} \in$ $\{1,2,3, \ldots\}[41]$.

\subsection{Channel estimation and causal ISI removal}

Our aim is to estimate the communication channel, $\boldsymbol{h}_{m}$, and based on that, estimate the transmitted symbols $\left\{s_{m}\right\}_{m \geq 1}$ according to the channel outputs $\left\{r_{m}\right\}_{m>1}$. We introduce a new adaptation method to obtain an accurate estimate of the causal part of the channel, denoted by $\hat{\boldsymbol{h}}_{m}$. Mathematically, the output of the estimated channel is $\hat{r}_{m}=\hat{\boldsymbol{h}}_{m}^{T} \boldsymbol{s}_{m}$, where $\boldsymbol{s}_{m} \triangleq\left[s_{m}, \ldots, s_{m-L+1}\right]^{T}$ is the vector of the current and past $L-1$ transmitted symbols. We use the error $e_{m}=r_{m}-\hat{r}_{m}$ to adapt the estimated channel after processing each sample, as shown in Fig. 2. As depicted in Fig. 2, $\boldsymbol{s}_{m}$ consists of the hard estimates of the transmitted symbols, i.e., in the training mode we use $s_{m}$, however, in the decision directed mode we use the quantized estimates $Q\left(\hat{s}_{m}\right)$, as the input to the channel estimator

As depicted in Fig. 2, we first remove the inter-symbol interference (ISI) effect generated by the causal part of the channel, i.e., $\boldsymbol{h}_{m}$, to obtain "cleaned" symbols $\tilde{r}_{m}$. We then use the past $N_{c}$ 
cleaned symbols at each time, in the causal part of the equalizer, to further reduce the ISI effect. Since the channel has a length of $L$, in order to obtain cleaned symbols $\tilde{r}_{m}$, we must remove the effect of the past $L-1$ symbols from each received symbol $r_{m}$, based on the estimated channel at time $m$. Therefore, inserting in a matrix form, we have

$\tilde{\boldsymbol{r}}_{m+1}=\boldsymbol{r}_{m+1}-\hat{\boldsymbol{H}}_{m}\left[s_{m-N_{c}-L+2}, \ldots, s_{m}\right]^{T}$,

where $\hat{\boldsymbol{H}}_{m}$ is the channel convolutional matrix defined as

$\hat{\boldsymbol{H}}_{m} \triangleq\left(\begin{array}{ccc}\hat{\boldsymbol{h}}_{m-N_{c}+1}^{T} & & \mathbf{0}_{N_{c}-1}^{T} \\ 0 & \hat{\boldsymbol{h}}_{m-N_{c}+2}^{T} & \mathbf{0}_{N_{c}-2}^{T} \\ \vdots & \ddots & \vdots \\ \mathbf{0}_{N_{c}-1}^{T} & & \hat{\boldsymbol{h}}_{m}^{T}\end{array}\right)_{N_{c} \times\left(N_{c}+L-1\right)}$

In addition, $\boldsymbol{r}_{m}=\left[r_{m-N_{c}+1}, \ldots, r_{m}\right]$ is the $N_{c} \times 1$ received signal vector, and $\tilde{\boldsymbol{r}}_{m}=\left[\tilde{r}_{m-N_{c}+1}, \ldots, \tilde{r}_{m}\right]$ is the cleaned version of the received signal vector $\left(\boldsymbol{r}_{m}\right)$ to be used as the input of the causal part of the equalizer.

In order to update the channel estimate, i.e., $\hat{\boldsymbol{h}}_{m}$, a cost function $C\left(e_{m}\right)$ is defined, e.g., in LMS method the cost is defined as $C\left(e_{m}\right)=E\left[\left|e_{m}\right|^{2}\right]$. Then, we derive an algorithm for updating $\hat{\boldsymbol{h}}_{m}$ based on minimization of this cost function using the stochastic gradient descent method [25]. Therefore, we update the tap weights vector as follows.

$\hat{\boldsymbol{h}}_{m+1}=\hat{\boldsymbol{h}}_{m}-\frac{1}{2} \mu \nabla_{\hat{\boldsymbol{h}}_{m}} \tilde{C}\left(e_{m}\right)$,

where $\nabla_{\hat{\boldsymbol{h}}_{m}} \tilde{C}\left(e_{m}\right)$ denotes an instantaneous approximation of the gradient of the cost function $C\left(e_{m}\right)$ with respect to $\hat{\boldsymbol{h}}_{m}$, i.e., the gradient obtained by removing the expectation and taking the gradient of the term inside [25]. As an example, in LMS method $C\left(e_{m}\right)=E\left[\left|e_{m}\right|^{2}\right]$, hence we update the tap weights vector of an LMS estimator as

$\hat{\boldsymbol{h}}_{m+1}=\hat{\boldsymbol{h}}_{m}+\mu e_{m} \boldsymbol{s}_{m}$,

where $\mu>0$ is the learning rate. Note that according to [25], for the LMS algorithm the learning rate (step size) should satisfy the following inequality:

$\mu \leq \frac{2}{\lambda_{\max }}$

where $\lambda_{\max }$ shows the maximum eigenvalue of the signal covariance matrix.

The updating expression in (6) is the well-known LMS update. However, we seek to provide a more robust updating algorithm through minimization of a different cost function. Note that the cost functions of the form $C\left(e_{m}\right)=E\left[\left|e_{m}\right|^{k}\right]$, which consist of only the $k$ th power of the error, either have a slow convergence, or do not perform well, from the stability viewpoint, in an impulsive noise environment [23]. Therefore, in Section 3, we use a logarithmic term in the cost function, which intrinsically introduces different powers of the error into the cost function. As a result, when an impulsive error occurs, the algorithm mitigates the effect of that sample in updating the equalizer coefficients by simply using the lower order norms of the error, whereas in impulsefree environments the algorithm accelerates the convergence using higher order norms of the error.

\subsection{Channel equalization}

To obtain the estimate $\hat{s}_{m}$, we use a linear channel equalizer, which is mathematically represented as $\hat{s}_{m}=\boldsymbol{w}_{m}^{T} \breve{\boldsymbol{r}}_{m}$, where $\check{\boldsymbol{r}}_{m} \triangleq\left[\tilde{r}_{m-N_{c}+1}, \ldots, \tilde{r}_{m}, r_{m+1}, \ldots, r_{m+N_{a}}\right]^{T}, N_{a}$ and $N_{c}$ represent the lengths of anti-causal and causal equalizers, respectively, and $\boldsymbol{w}_{m} \triangleq\left[w_{-N_{c}+1, m}, \ldots, w_{N_{a}, m}\right]^{T}$ is the tap weights vector of the linear equalizer at time $m$. We define the error in estimating the transmitted symbol $s_{m}$ as $\epsilon_{m}=s_{m}-\hat{s}_{m}$. We then use the conventional normalized LMS (NLMS) method to update the equalizer. In the rest of the paper, we focus on the channel estimation part of the system.

\section{Adaptive channel estimation based on logarithmic cost functions}

In this section, we explain our method mathematically and introduce two channel estimators based on the logarithmic costs [23]. Thus, we define the new cost as

$C\left(e_{m}\right) \triangleq \Phi\left(e_{m}\right)-\frac{1}{a} \ln \left(1+a \Phi\left(e_{m}\right)\right)$,

where $a>0$ is a design parameter. From the properties of the logarithmic function [23], we deduce that when $a \Phi\left(e_{m}\right) \ll 1$

$C\left(e_{m}\right) \rightarrow \frac{a}{2} \Phi^{2}\left(e_{m}\right)-\frac{a^{2}}{3} \Phi^{3}\left(e_{m}\right)+\ldots$

and,

$C\left(e_{m}\right) \rightarrow \Phi\left(e_{m}\right), \quad$ as $e_{m} \rightarrow \pm \infty$.

It is straightforward to show that the new cost function $C\left(e_{m}\right)$ is a convex function of $e_{m}$, if the cost function $\Phi\left(e_{m}\right)$ is convex. Therefore, in the new algorithm, we seek to minimize a cost function that is mainly consisted of the first and second powers of the primary cost function $\Phi\left(e_{m}\right)$, based on the error amount.

\subsection{First order methods}

In this section, we use first order methods to adaptively adjust the channel estimate $\hat{\boldsymbol{h}}_{m}$. For this purpose, we use the stochastic gradient method [25] to derive a recursion expression for updating $\hat{\boldsymbol{h}}_{m}$. This yields,

$$
\begin{aligned}
\hat{\boldsymbol{h}}_{m+1} & =\hat{\boldsymbol{h}}_{m}-\frac{1}{2} \mu \nabla_{\hat{\boldsymbol{h}}_{m}} C\left(e_{m}\right) \\
& =\hat{\boldsymbol{h}}_{m}-\mu \frac{a \Phi\left(e_{m}\right)}{1+a \Phi\left(e_{m}\right)}\left[\nabla_{\hat{\boldsymbol{h}}_{m}} \Phi\left(e_{m}\right)\right] .
\end{aligned}
$$

Particularly, in this framework, we adopt a well-known cost function, e.g., $\Phi\left(e_{m}\right)=E\left[\left|e_{m}\right|^{2}\right]$, as the primary cost function $\Phi\left(e_{m}\right)$. Suppose that $\Phi\left(e_{m}\right)$ is the expectation of another function $\varphi\left(e_{m}\right)$, i.e., $\Phi\left(e_{m}\right)=E\left[\varphi\left(e_{m}\right)\right]$. Then, using the instantaneous approximation for $\Phi\left(e_{m}\right)$ [25], the general stochastic gradient update is given by

$\hat{\boldsymbol{h}}_{m+1}=\hat{\boldsymbol{h}}_{m}+\mu \frac{a \varphi\left(e_{m}\right)}{1+a \varphi\left(e_{m}\right)}\left[\nabla_{e_{m}} \varphi\left(e_{m}\right)\right] \boldsymbol{s}_{m}$.

We present two channel estimation methods based on the introduced approach. We then show the superior performance of these methods through highly realistic experiments in Section 5.

1. The logarithmic cost least mean squares (LCLMS):

Here, we adopt $\varphi\left(e_{m}\right)=\left|e_{m}\right|^{2}$, which (according to (8)) yields the following update on the tap weights vector

$$
\begin{aligned}
\hat{\boldsymbol{h}}_{m+1} & =\hat{\boldsymbol{h}}_{m}+\mu \frac{a\left|e_{m}\right|^{2}}{1+a\left|e_{m}\right|^{2}}\left[2 e_{m}\right] \boldsymbol{s}_{m}^{*} \\
& =\hat{\boldsymbol{h}}_{m}+\mu^{\prime} \frac{e_{m}\left|e_{m}\right|^{2}}{1+a\left|e_{m}\right|^{2}} \boldsymbol{s}_{m}^{*} .
\end{aligned}
$$


2. The logarithmic cost least mean absolutes (LCLMA):

In this case, we adopt $\varphi\left(e_{m}\right)=\left|e_{m}\right|$, and according to (8), obtain the following update on the tap weights vector,

$\hat{\boldsymbol{h}}_{m+1}=\hat{\boldsymbol{h}}_{m}+\mu \frac{a\left|e_{m}\right|}{1+a\left|e_{m}\right|}\left[\operatorname{csgn}\left(e_{m}\right)\right] \boldsymbol{s}_{m}^{*}$

where $\operatorname{csgn}\left(e_{m}\right)=\frac{1}{\sqrt{2}}\left(\operatorname{sign}\left(\operatorname{Re}\left(e_{m}\right)\right)+j \operatorname{sign}\left(\operatorname{Im}\left(e_{m}\right)\right)\right)$, like in [39].

As shown in the simulations, the LCLMS algorithm results in an improved convergence speed over the conventional LMS algorithm, while achieving the comparable stability to LMS method. Similarly, we achieve an improved convergence speed performance over the conventional SA algorithm by using LCLMA algorithm, while preserving the robustness against impulsive noise. Hence, these are elegant alternatives to the conventional methods for UWA channel estimation.

\subsection{Second order methods}

By defining the cost function as $J_{m} \triangleq \sum_{i=0}^{m} \lambda^{m-i} C\left(e_{i}\right)$, we seek to update $\hat{\boldsymbol{h}}_{m}$ in order to minimize $J_{m}$. Therefore, by solving

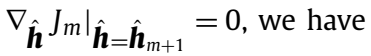

$$
\begin{aligned}
\nabla_{\hat{\boldsymbol{h}}^{J}} & =\sum_{i=0}^{m} \lambda^{m-i} \nabla_{\hat{\boldsymbol{h}} C\left(e_{i}\right)} \\
& =\sum_{i=0}^{m} \lambda^{m-i} \frac{\partial C\left(e_{i}\right)}{\partial \varphi\left(e_{i}\right)} \frac{\partial \varphi\left(e_{i}\right)}{\partial e_{i}} \nabla_{\hat{\boldsymbol{h}}} e_{i} \\
& =\sum_{i=0}^{m} \lambda^{m-i} \frac{a \varphi\left(e_{i}\right)}{1+a \varphi\left(e_{i}\right)} \frac{\partial \varphi\left(e_{i}\right)}{\partial e_{i}}\left(-\boldsymbol{s}_{i}\right) \\
& =\sum_{i=0}^{m} \lambda^{m-i} \frac{a \frac{\varphi\left(e_{i}\right)}{e_{i}}}{1+a \varphi\left(e_{i}\right)} \frac{\partial \varphi\left(e_{i}\right)}{\partial e_{i}}\left(-\boldsymbol{s}_{i}\right) e_{i} \\
& =\sum_{i=0}^{m} \lambda^{m-i} w\left(e_{i}\right)\left(-\boldsymbol{s}_{i}\right)\left(r_{i}-\boldsymbol{s}_{i}^{H} \hat{\boldsymbol{h}}\right),
\end{aligned}
$$

where $w\left(e_{i}\right)=\frac{a \frac{\varphi\left(e_{i}\right)}{e_{i}}}{1+a \varphi\left(e_{i}\right)} \frac{\partial \varphi\left(e_{i}\right)}{\partial e_{i}}$ is considered as the weight of the $i$ th sample. Finally, the equation $\left.\nabla_{\hat{\boldsymbol{h}}} J_{m}\right|_{\hat{\boldsymbol{h}}=\hat{\boldsymbol{h}}_{m+1}}=0$ yields the following equation for $\hat{\boldsymbol{h}}_{m+1}$

$\sum_{i=0}^{m} \lambda^{m-i} w\left(e_{i}\right) \boldsymbol{s}_{i} \boldsymbol{s}_{i}^{H} \hat{\boldsymbol{h}}_{m+1}=\sum_{i=0}^{m} \lambda^{m-i} w\left(e_{i}\right) \boldsymbol{s}_{i} r_{i}$.

Hence [24]

$\hat{\boldsymbol{h}}_{m+1}=\boldsymbol{\Omega}_{m}^{-1} \boldsymbol{\psi}_{m}$,

where $\boldsymbol{\Omega}_{m}=\sum_{i=0}^{m} \lambda^{m-i} w\left(e_{i}\right) \boldsymbol{s}_{i} \boldsymbol{s}_{i}^{T}$ and $\boldsymbol{\psi}_{m}=\sum_{i=0}^{m} \lambda^{m-i} w\left(e_{i}\right) \boldsymbol{s}_{i} r_{i}$. We also observe that

$\boldsymbol{\Omega}_{m}=\lambda \boldsymbol{\Omega}_{m-1}+w\left(e_{m}\right) \boldsymbol{s}_{m} \boldsymbol{s}_{m}^{H}$,

and

$\boldsymbol{\psi}_{m}=\lambda \boldsymbol{\psi}_{m-1}+w\left(e_{m}\right) \boldsymbol{s}_{m} r_{m}$.

Thus, by using the matrix inversion lemma [25], $\boldsymbol{\Omega}_{m}^{-1}$ can be calculated as follows.

$\boldsymbol{\Omega}_{m}^{-1}=\lambda^{-1}\left(\boldsymbol{\Omega}_{m-1}^{-1}-\frac{1}{\frac{\lambda}{w\left(e_{m}\right)}+\boldsymbol{s}_{m}^{H} \boldsymbol{\Omega}_{m-1}^{-1} \boldsymbol{s}_{m}} \boldsymbol{\Omega}_{m-1}^{-1} \boldsymbol{s}_{m} \boldsymbol{s}_{m}^{H} \boldsymbol{\Omega}_{m-1}^{-1}\right)$.
Now by defining $\boldsymbol{P}_{m} \triangleq \boldsymbol{\Omega}_{m}^{-1}$, and $\boldsymbol{g}_{m} \triangleq \frac{w\left(e_{m}\right) \boldsymbol{P}_{m-1} \boldsymbol{s}_{m}}{\lambda+w\left(e_{m}\right) \boldsymbol{S}_{m}^{H} \boldsymbol{P}_{m-1} \boldsymbol{s}_{m}}$, we have

$\boldsymbol{P}_{m}=\lambda^{-1}\left(\boldsymbol{P}_{m-1}-\boldsymbol{g}_{m} \boldsymbol{s}_{m}^{H} \boldsymbol{P}_{m-1}\right)$,

and rearranging the terms in the definition of $\boldsymbol{g}_{m}$ yields

$$
\begin{aligned}
\boldsymbol{g}_{m} & =\frac{w\left(e_{m}\right)}{\lambda}\left[\boldsymbol{P}_{m-1}-\boldsymbol{g}_{m} \boldsymbol{s}_{m}^{H} \boldsymbol{P}_{m-1}\right] \boldsymbol{s}_{m} \\
& =w\left(e_{m}\right) \boldsymbol{P}_{m} \boldsymbol{s}_{m} .
\end{aligned}
$$

Substituting (11) and (12) in the expression $\hat{\boldsymbol{h}}_{m+1}=\boldsymbol{P}_{m} \boldsymbol{\psi}_{m}$ and expanding it results in

$$
\begin{aligned}
\hat{\boldsymbol{h}}_{m+1} & =\lambda \boldsymbol{P}_{m} \boldsymbol{\psi}_{m-1}+w\left(e_{m}\right) \boldsymbol{P}_{m} \boldsymbol{s}_{m} r_{m} \\
& =\left(\boldsymbol{P}_{m-1}-\boldsymbol{g}_{m} \boldsymbol{s}_{m}^{H} \boldsymbol{P}_{m-1}\right) \boldsymbol{\psi}_{m-1}+\boldsymbol{g}_{m} r_{m} \\
& =\hat{\boldsymbol{h}}_{m}+\boldsymbol{g}_{m}\left(r_{m}-\boldsymbol{s}_{m}^{H} \hat{\boldsymbol{h}}_{m}\right) .
\end{aligned}
$$

Hence, the final second order updating algorithm is

$e_{m}=r_{m}-\boldsymbol{s}_{m}^{H} \hat{\boldsymbol{h}}_{m}$,

$\boldsymbol{g}_{m}=\frac{w\left(e_{m}\right) \boldsymbol{P}_{m-1} \boldsymbol{s}_{m}}{\lambda+w\left(e_{m}\right) \boldsymbol{s}_{m}^{H} \boldsymbol{P}_{m-1} \boldsymbol{s}_{m}}$

$\hat{\boldsymbol{h}}_{m+1}=\hat{\boldsymbol{h}}_{m}+e_{m} \mathbf{g}_{m}$,

$\boldsymbol{P}_{m}=\lambda^{-1}\left(\boldsymbol{P}_{m-1}-\boldsymbol{g}_{m} \boldsymbol{s}_{m}^{H} \boldsymbol{P}_{m-1}\right)$,

where $\boldsymbol{P}_{0}=v^{-1} \boldsymbol{I}$, and $0<v \ll 1$. We point out that for the $\varphi\left(e_{m}\right)=\left|e_{m}\right|^{2}$, we have $w\left(e_{m}\right)=\frac{2 a\left|e_{m}\right|^{2}}{1+a\left|e_{m}\right|^{2}}$. However, according to (9) multiplying the $w\left(e_{m}\right)$ by a constant term does not affect the algorithm. Therefore, we use $w\left(e_{m}\right)=\frac{\left|e_{m}\right|^{2}}{1+a\left|e_{m}\right|^{2}}$ in (15) to obtain the logarithmic cost recursive least squares (LCRLS) algorithm. Moreover, by using $\varphi\left(e_{m}\right)=\left|e_{m}\right|$, we achieve $w\left(e_{m}\right)=\frac{1}{1+a\left|e_{m}\right|}$, which results in the logarithmic cost recursive least absolutes (LCRLA) algorithm.

\section{Performance analysis}

In this section we provide a thorough analysis for the mean square error (MSE) performance of the proposed methods. Since the channel is highly time-varying, we use the notion of excess MSE (EMSE) as in [25]. Furthermore, in order to be more realistic, we assume carrier frequency offset as well as impulsive ambient noise. We first present the impulsive noise model used in the analyses, then we establish our analyses based on the widely used assumptions in the literature. Note that although for the sake of notational simplicity, we assume $a=1$ in all algorithms, the results can be readily extended to other values of $a$.

In order to analyze the tracking performance of the introduced algorithms, we assume a random walk model [25] for the discrete channel vector $\boldsymbol{h}_{m}$ that yields the minimum mean squared error such that

$$
\begin{aligned}
\boldsymbol{h}_{m} & =\boldsymbol{h}+\boldsymbol{\theta}_{m}, \\
\boldsymbol{\theta}_{m+1} & =\alpha \boldsymbol{\theta}_{m}+\boldsymbol{q}_{m} .
\end{aligned}
$$

Moreover, due to the carrier offsets, we consider the following model for the received data

$r_{m}=\boldsymbol{s}_{m}^{H} \boldsymbol{h}_{m} e^{j \Omega m}+n_{m}$.

We define $\tilde{\boldsymbol{h}}_{m} \triangleq \boldsymbol{h}_{m} e^{j \Omega m}-\hat{\boldsymbol{h}}_{m}$ and $\boldsymbol{q}_{m} \in \mathbb{R}^{L}$ is a zero-mean vector process with covariance matrix $E\left[\boldsymbol{q}_{m} \boldsymbol{q}_{m}^{H}\right]=\boldsymbol{Q}$.

MSE $=\sigma_{n}^{2}+\lim _{m \rightarrow \infty}\left|\boldsymbol{s}_{m}^{H} \tilde{\boldsymbol{h}}_{m}\right|^{2}$.

We define the EMSE as 
$\zeta \triangleq \lim _{m \rightarrow \infty}\left|\boldsymbol{s}_{m}^{H} \tilde{\boldsymbol{h}}_{m}\right|^{2}=\lim _{m \rightarrow \infty}\left|e_{a, m}\right|^{2}$.

We next introduce the impulsive noise model we use in our analyses.

Impulsive noise model: Since the received signal is subjected to impulsive noise, we model the estimation noise as a summation of two independent random terms $[42,43]$ as

$n_{m}=n_{g, m}+z_{m} n_{i, m}$,

where $n_{g, m}$ is the ordinary AWGN noise signal that is zero-mean Gaussian with variance $\sigma_{g}^{2}$ and $n_{i, m}$ is the impulsive part of the noise that is also a zero-mean Gaussian random process with a significantly large variance $\sigma_{i}^{2}$. Here, $z_{m}$ is generated through a Bernoulli random process and determines the occurrence of the impulses in the noise signal with $p_{Z}\left(z_{m}=1\right)=v_{i}$ and $p_{Z}\left(z_{m}=\right.$ $0)=1-v_{i}$, where $v_{i}$ is the frequency of the impulses. The overall probability density function of the noise signal $n_{m}$ is given by

$p_{n}\left(n_{m}\right)=\frac{1-v_{i}}{\sqrt{2 \pi} \sigma_{g}} \exp \left(-\frac{n_{m}^{2}}{2 \sigma_{g}^{2}}\right)+\frac{v_{i}}{\sqrt{2 \pi} \sigma_{n}} \exp \left(-\frac{n_{m}^{2}}{2 \sigma_{n}^{2}}\right)$,

where $\sigma_{n}^{2}=\sigma_{g}^{2}+\sigma_{i}^{2}$.

\subsection{MSE analysis of the first order methods}

Assuming that $\boldsymbol{q}_{m}$ is independent from the received and noise signals, at the steady state, we have the following general variance relation for an adaptive filter with the error nonlinearity $g\left(e_{m}\right)$ $[25,39]$,

$$
\begin{aligned}
2 \operatorname{Re}\{ & \left.E\left[e_{a, m}^{*} g\left(e_{m}\right)\right]\right\} \\
= & \mu E\left[\left\|\boldsymbol{s}_{m}\right\|^{2}\left|g\left(e_{m}\right)\right|^{2}\right]+\mu^{-1} \operatorname{Tr}(\boldsymbol{Q}) \\
& +\mu^{-1}\left|1-e^{j \Omega}\right|^{2}\|\boldsymbol{h}\|^{2}+\mu^{-1}\left|1-\alpha e^{j \Omega}\right|^{2} \operatorname{Tr}(\boldsymbol{\Theta}) \\
& -2 \mu^{-1} \operatorname{Re}\left\{\left(1-e^{-j \Omega}\right) \boldsymbol{h} E\right. \\
& \left.\times\left[\left(\tilde{\boldsymbol{h}}_{m-1}-\mu \boldsymbol{s}_{m}^{*} g\left(e_{m}\right)\right) e^{-j \Omega(m-1)}\right]\right\} \\
& -2 \mu^{-1} \operatorname{Re}\left\{\left(1-\alpha^{*} e^{-j \Omega}\right) \boldsymbol{h} E\right. \\
& \left.\times\left[\boldsymbol{\theta}_{m-1}^{*}\left(\tilde{\boldsymbol{h}}_{m-1}-\mu \boldsymbol{s}_{m}^{*} g\left(e_{m}\right)\right) e^{-j \Omega(m-1)}\right]\right\},
\end{aligned}
$$

where

$\boldsymbol{\Theta} \triangleq \lim _{m \rightarrow \infty} E\left[\boldsymbol{\theta}_{m} \boldsymbol{\theta}_{m}^{*}\right]=\frac{1}{1-|\alpha|^{2}} \boldsymbol{Q}$,

and $g\left(e_{m}\right)$ is the nonlinear error function [44]. Moreover, $e_{m}=$ $e_{a, m}+n_{m}$, in which $e_{a, m}=\boldsymbol{s}_{m} \tilde{\boldsymbol{h}}_{m}$ is a priori estimation error and $n_{m}$ is the estimation noise, i.e., the error resulted from the optimal linear estimator. For the proposed algorithms, $g\left(e_{m}\right)$ is defined as

$g\left(e_{m}\right) \triangleq \frac{\partial \varphi\left(e_{m}\right)}{\partial e_{m}} \frac{\varphi\left(e_{m}\right)}{1+\varphi\left(e_{m}\right)}$

We note that in an impulse-free environment, i.e., when $e_{m} \ll 1$, we have

$$
\frac{a \varphi\left(e_{m}\right)}{1+a \varphi\left(e_{m}\right)} \approx a \varphi\left(e_{m}\right)
$$

On the other hand, in an impulsive noise environment we have

$$
\frac{a \varphi\left(e_{m}\right)}{1+a \varphi\left(e_{m}\right)} \approx 1
$$

Therefore, we decompose the left hand side of (20) as

$$
\begin{aligned}
E\left[\left|e_{a, m}\right|^{2}\right]= & E\left[\left|e_{a, m}\right|^{2} \mid z_{m}=1\right] p_{Z}\left(z_{m}=1\right) \\
& +E\left[\left|e_{a, m}\right|^{2} \mid z_{m}=0\right] p_{Z}\left(z_{m}=0\right) \\
= & E\left[\left|e_{a, m}\right|^{2} \mid g\left(e_{m}\right)=\frac{\partial \varphi\left(e_{m}\right)}{\partial e_{m}}\right] v_{i} \\
& +E\left[\left|e_{a, m}\right|^{2} \mid g\left(e_{m}\right)=\frac{\partial \varphi\left(e_{m}\right)}{\partial e_{m}} \varphi\left(e_{m}\right)\right]\left(1-v_{i}\right),
\end{aligned}
$$

and we also note that

$$
\begin{aligned}
& E\left[e_{a, m}^{*} g\left(e_{m}\right) \mid z_{m}=1\right]=E\left[e_{a, m}^{*} \frac{\partial \varphi(e(t))}{\partial e(t)}\right], \\
& E\left[e_{a, m}^{*} g\left(e_{m}\right) \mid z_{m}=0\right]=E\left[e_{a, m}^{*} \frac{\partial \varphi(e(t))}{\partial e(t)} \varphi(e(t))\right] .
\end{aligned}
$$

We use (25) to calculate the terms on the right hand side of (24). We also note that for the LCLMS and LCLMA methods, $\varphi\left(e_{m}\right)=$ $\left|e_{m}\right|^{2}$ and $\varphi\left(e_{m}\right)=\left|e_{m}\right|$, respectively. In our analysis, we use the following assumptions:

Assumption 1. The noise signal $n_{m}$ is a zero-mean, independently, and identically distributed (i.i.d.), Gaussian random variable, and independent from $\boldsymbol{s}_{m}$. The transmitted signal $\boldsymbol{s}_{m}$ is also a zeromean i.i.d. Gaussian random variable with the auto-correlation ma$\operatorname{trix} \boldsymbol{R}_{\boldsymbol{s}} \triangleq E\left[\boldsymbol{s}_{m} \boldsymbol{s}_{m}^{H}\right]$.

Assumption 2. The a priori estimation error $e_{a, m}$ has Gaussian distribution. This assumption is reasonable by the Assumption 1, whenever $L_{c}$ is sufficiently large and the learning rate $\mu$ is sufficiently small [44].

Assumption 3. The random processes $\left\|\boldsymbol{s}_{m}\right\|_{\boldsymbol{P}_{m}}^{2}$ and $g^{2}\left(e_{m}\right)$ are uncorrelated, which results in the following separation

$E\left[\left\|\boldsymbol{s}_{m}\right\|_{\boldsymbol{P}_{m}}^{2} g^{2}\left(e_{m}\right)\right]=E\left[\left\|\boldsymbol{s}_{m}\right\|_{\boldsymbol{P}_{m}}^{2}\right] E\left[g^{2}\left(e_{m}\right)\right]$.

Based on (20), Assumptions 1-3, and the Price's Theorem [25] for $E\left[e_{a, m}^{*} \operatorname{csgn}\left(e_{m}\right)\right]$, it can be straightforwardly shown that $[25$, 39]

$$
\begin{aligned}
E\left[\left|e_{a, m}\right|^{2} \mid g\left(e_{m}\right)=\operatorname{csgn}\left(e_{m}\right)\right] & =\zeta^{\mathrm{SA}} \\
E\left[\left|e_{a, m}\right|^{2} \mid g\left(e_{m}\right)=e_{m}\right] & =\zeta^{\mathrm{LMS}} \\
E\left[\left|e_{a, m}\right|^{2} \mid g\left(e_{m}\right)=e_{m}^{3}\right] & =\zeta^{\mathrm{LMF}} .
\end{aligned}
$$

However, in [39], the excess MSE for SA, LMS, and LMF methods are calculated as follows,

$$
\begin{aligned}
\zeta^{\mathrm{LCLMA}}= & v_{i} \zeta^{\mathrm{SA}}+\left(1-v_{i}\right) \zeta^{\mathrm{LMS}} \\
= & v_{i} \frac{2 \mu \operatorname{Tr}(\boldsymbol{R} \boldsymbol{s})+\mu^{-1} \beta(\eta)}{2 \eta} \\
& +\left(1-v_{i}\right) \frac{\mu \sigma_{g}^{2} \operatorname{Tr}(\boldsymbol{R} \boldsymbol{s})+\mu^{-1} \beta(1)}{2},
\end{aligned}
$$

where

$$
\begin{aligned}
\eta= & \frac{2}{\sqrt{\pi\left(\zeta^{S A}+\sigma_{n}^{2}\right)}}, \\
\beta(\gamma)= & \left|1-e^{j \Omega}\right|^{2} \operatorname{Re}\left\{\operatorname{Tr}\left(\left(\boldsymbol{I}-2\left(\boldsymbol{X}_{\gamma}-\mu \boldsymbol{R}_{\boldsymbol{S}}\right)\right) \boldsymbol{h} \boldsymbol{h}^{H}\right)\right\} \\
& +\left|1-\alpha e^{j \Omega}\right|^{2} \operatorname{Re}\left\{\operatorname{Tr}\left(\left(\boldsymbol{I}-2\left(\alpha^{*} \boldsymbol{X}_{\gamma, \alpha}-\mu \boldsymbol{R}_{\boldsymbol{S}}\right)\right) \boldsymbol{\Theta}\right)\right\} \\
& +\operatorname{Re}\left\{\operatorname{Tr}\left(\left(\boldsymbol{I}+2\left(e^{j \Omega}-\alpha^{*}\right) \boldsymbol{X}_{\alpha}\right) \boldsymbol{Q}\right)\right\},
\end{aligned}
$$

and for $\forall \gamma$ 


$$
\begin{aligned}
\boldsymbol{X}_{\gamma} & =\left(\boldsymbol{I}-\mu \gamma \boldsymbol{R}_{\boldsymbol{s}}\right)\left[\boldsymbol{I}-e^{j \Omega}\left(\boldsymbol{I}-\mu \gamma \boldsymbol{R}_{\boldsymbol{S}}\right)^{-1}\right]^{-1}, \\
\boldsymbol{X}_{\gamma, \alpha} & =\left(\boldsymbol{I}-\mu \gamma \boldsymbol{R}_{\boldsymbol{s}}\right)\left[\alpha^{*} \boldsymbol{I}-e^{j \Omega}\left(\boldsymbol{I}-\mu \gamma \boldsymbol{R}_{\boldsymbol{S}}\right)^{-1}\right]^{-1} .
\end{aligned}
$$

Similarly, we obtain the following results for the excess MSE of the LCLMS method.

$$
\begin{aligned}
\zeta^{\mathrm{LCLMS}}= & v_{i} \zeta^{\mathrm{LMS}}+\left(1-v_{i}\right) \zeta^{\mathrm{LMF}} \\
= & v_{i} \frac{\mu \sigma_{n}^{2} \operatorname{Tr}(\boldsymbol{R} \boldsymbol{s})+\mu^{-1} \beta(1)}{2} \\
& +\left(1-v_{i}\right) \frac{\mu \xi^{6} \operatorname{Tr}(\boldsymbol{R} \boldsymbol{s})+\mu^{-1} \beta\left(3 \sigma_{g}^{2}\right)}{6 \sigma_{g}^{2}},
\end{aligned}
$$

where

$\xi^{6} \triangleq E\left[\left|n_{g, m}\right|^{6}\right]$.

\subsection{MSE analysis of the second order methods}

Here, we use the same assumptions as the first order analyses. According to (13), and defining $g\left(e_{m}\right) \triangleq e_{m} w\left(e_{m}\right)$, we have the recursion $\hat{\boldsymbol{h}}_{m+1}=\hat{\boldsymbol{h}}_{m}+g\left(e_{m}\right) \boldsymbol{P}_{m} \boldsymbol{s}_{m}$. Thus,

$$
\tilde{\boldsymbol{h}}_{m+1}=\tilde{\boldsymbol{h}}_{m}-\boldsymbol{P}_{m} \boldsymbol{s}_{m} g\left(e_{m}\right)+\boldsymbol{c}_{m} e^{j \Omega m},
$$

which yields the following relation between the a priori and a posteriori error estimations

$$
\begin{aligned}
e_{p, m} & =\boldsymbol{s}_{m}^{H}\left(\tilde{\boldsymbol{h}}_{m+1}-\boldsymbol{c}_{m} e^{j \Omega m}\right) \\
& =e_{a, m}-\left\|\boldsymbol{s}_{m}\right\|_{\boldsymbol{P}_{m}}^{2} g\left(e_{m}\right) .
\end{aligned}
$$

Equivalently, we observe that

$\tilde{\boldsymbol{h}}_{m+1}-\boldsymbol{c}_{m} e^{j \Omega m}=\tilde{\boldsymbol{h}}_{m}-\boldsymbol{P}_{m} \boldsymbol{s}_{m} \frac{e_{a, m}-e_{p, m}}{\left\|\boldsymbol{s}_{m}\right\|_{\boldsymbol{P}_{m}}^{2}}$.

Therefore, we have

$$
\left\|\tilde{\boldsymbol{h}}_{m+1}-\boldsymbol{c}_{m} e^{j \Omega m}\right\|_{\boldsymbol{P}_{m}^{-1}}^{2}=\left\|\tilde{\boldsymbol{h}}_{m}\right\|_{\boldsymbol{P}_{m}^{-1}}^{2}+\frac{\left|e_{p, m}\right|^{2}-\left|e_{a, m}\right|^{2}}{\left\|\boldsymbol{s}_{m}\right\|_{\boldsymbol{P}_{m}}^{2}} .
$$

Assuming that $\boldsymbol{P}_{m}^{-1}$ converges to its mean value, $E\left[\boldsymbol{P}_{m}^{-1}\right]=\boldsymbol{P}^{-1}$, in the steady state, we have $E\left[\left\|\tilde{\boldsymbol{h}}_{m+1}\right\|_{\boldsymbol{P}_{m+1}^{-1}}^{2}\right]=E\left[\left\|\tilde{\boldsymbol{h}}_{m}\right\|_{\boldsymbol{P}_{m}^{-1}}^{2}\right]$ [39], which results in

$$
\begin{aligned}
E\left[e_{a, m}^{*} g\left(e_{m}\right)\right]= & E\left[\left\|\boldsymbol{s}_{m}\right\|_{\boldsymbol{P}_{m}}^{2}\left|g\left(e_{m}\right)\right|^{2}\right]+\left\|\boldsymbol{c}_{m}\right\|_{\boldsymbol{P}_{m}^{-1}}^{2} \\
& +2 \operatorname{Re}\left\{E\left[e^{-j \Omega m} \boldsymbol{c}_{m}^{H} \boldsymbol{P}_{m}^{-1}\left(\tilde{\boldsymbol{h}}_{m}-\boldsymbol{P}_{m} \boldsymbol{s}_{m} g\left(e_{m}\right)\right)\right]\right\} .
\end{aligned}
$$

We next investigate three cases: $g\left(e_{m}\right)=e_{m}, g\left(e_{m}\right)=e_{m}\left|e_{m}\right|^{2}$ and $g\left(e_{m}\right)=\operatorname{sign}\left(e_{m}\right)$. We define $\boldsymbol{v}_{m} \triangleq E\left[\boldsymbol{P}_{m}^{-1} \tilde{\boldsymbol{h}}_{m}\right]$ and $\boldsymbol{\Sigma} \triangleq$ $E\left[\boldsymbol{P}_{m}^{-1} \tilde{\boldsymbol{h}}_{m} \boldsymbol{\theta}_{m}^{H}\right]$. In addition, we assume that in the steady state, $\boldsymbol{P}_{m}$ is independent of $\tilde{\boldsymbol{h}}_{m}$ and $\boldsymbol{c}_{m}$.

1. For $g\left(e_{m}\right)=e_{m}$,

$$
\begin{aligned}
& \boldsymbol{v}_{m+1}=\left(\boldsymbol{I}-\boldsymbol{R}_{\boldsymbol{S}}\right) \boldsymbol{v}_{m}+\boldsymbol{P}^{-1} \boldsymbol{h}\left(e^{j \Omega}-1\right) e^{j \Omega m}, \\
& \boldsymbol{\Sigma}_{m+1}=\alpha\left(\boldsymbol{I}-\boldsymbol{R}_{\boldsymbol{S}}\right) \boldsymbol{\Sigma}_{m}-\boldsymbol{P}^{-1} \boldsymbol{C} e^{j \Omega m} .
\end{aligned}
$$

With a method similar to [39], we obtain

$$
\begin{aligned}
E\left[\boldsymbol{P}_{m}^{-1} \tilde{\boldsymbol{h}}_{m}\right] & =\boldsymbol{v} e^{j \Omega m}, \\
E\left[\boldsymbol{P}_{m}^{-1} \tilde{\boldsymbol{h}}_{m} \boldsymbol{\theta}_{m}^{T}\right] & =\boldsymbol{\Sigma} e^{j \Omega m},
\end{aligned}
$$

where

$$
\begin{aligned}
\boldsymbol{v} & =\left[\boldsymbol{I}-\boldsymbol{R}_{\boldsymbol{S}}-e^{j \Omega} \boldsymbol{I}\right]^{-1} \boldsymbol{P}^{-1} \boldsymbol{h}\left(1-e^{j \Omega}\right), \\
\boldsymbol{\Sigma} & =\left[\alpha\left(\boldsymbol{I}-\boldsymbol{R}_{\boldsymbol{S}}\right)-e^{j \Omega} \boldsymbol{I}\right]^{-1} \boldsymbol{P}^{-1} \boldsymbol{C},
\end{aligned}
$$

and

$\boldsymbol{C}=\alpha^{*}\left[1-\alpha e^{j \Omega}\right] \boldsymbol{\Theta}-e^{j \Omega} \boldsymbol{Q}$,

which yields the following expression for the excess MSE

$$
\begin{aligned}
{[1-} & \left.\operatorname{Tr}\left(\boldsymbol{R}_{\boldsymbol{S}} \boldsymbol{P}\right)\right] \zeta_{e_{m}} \\
= & \operatorname{Tr}\left(\boldsymbol{P}^{-1} \boldsymbol{Q}\right)+\left|1-e^{j \Omega}\right|^{2}\|\boldsymbol{h}\|^{2}+\left|1-\alpha e^{j \Omega}\right|^{2} \operatorname{Tr}\left(\boldsymbol{P}^{-1} \boldsymbol{\Theta}\right) \\
& -2 \operatorname{Re}\left\{\left(1-e^{-j \Omega}\right) \boldsymbol{h}^{T}(\boldsymbol{I}-\boldsymbol{R} \boldsymbol{S}) \boldsymbol{v}\right. \\
& \left.+\operatorname{Tr}\left[\left(1-\alpha^{*} e^{-j \Omega}\right)\left(\boldsymbol{I}-\boldsymbol{R}_{\boldsymbol{S}}\right) \boldsymbol{\Sigma}\right]\right\}
\end{aligned}
$$

2. For $g\left(e_{m}\right)=e_{m}\left|e_{m}\right|^{2}$.

Since in our final analysis, this case appears when the noise is Gaussian (non-impulsive), we assume that the noise power is $\sigma_{g}^{2}$. Furthermore, we use the assumption $\left|e_{a, m}\right|^{2} \ll \sigma_{g}^{2}$ [39], hence by substituting $g\left(e_{m}\right)$ in (36) and simplifying the result, we obtain the following expression for EMSE:

$$
\begin{aligned}
& {\left[3 \sigma_{g}^{2}-15 \xi^{4} \operatorname{Tr}\left(\boldsymbol{R}_{\boldsymbol{S}} \boldsymbol{P}\right)\right] \zeta_{e_{m}\left|e_{m}\right|^{2}}} \\
& \quad=\xi^{6} \operatorname{Tr}\left(\boldsymbol{R}_{\boldsymbol{S}} \boldsymbol{P}\right)+\operatorname{Tr}\left(\boldsymbol{P}^{-1} \boldsymbol{Q}\right) \\
& \quad+\left|1-e^{j \Omega}\right|^{2}\|\boldsymbol{h}\|^{2}+\left|1-\alpha e^{j \Omega}\right|^{2} \operatorname{Tr}\left(\boldsymbol{P}^{-1} \boldsymbol{\Theta}\right) .
\end{aligned}
$$

3. For $g\left(e_{m}\right)=\operatorname{csgn}\left(e_{m}\right)$.

By using the price theorem, we have

$$
\begin{aligned}
\eta \zeta_{\operatorname{csgn}}\left(e_{m}\right) \\
=\operatorname{Tr}\left(\boldsymbol{R}_{\boldsymbol{S}} \boldsymbol{P}\right)+\operatorname{Tr}\left(\boldsymbol{P}^{-1} \boldsymbol{Q}\right)+\left|1-e^{j \Omega}\right|^{2}\|\boldsymbol{h}\|^{2} \\
\quad+\left|1-\alpha e^{j \Omega}\right|^{2} \operatorname{Tr}\left(\boldsymbol{P}^{-1} \boldsymbol{\Theta}\right) \\
\quad-2 \operatorname{Re}\left\{\left(1-e^{-j \Omega}\right) \boldsymbol{h}^{T}\left(\boldsymbol{I}-\eta \boldsymbol{R}_{\boldsymbol{S}}\right) \boldsymbol{v}\right. \\
\left.\quad+\operatorname{Tr}\left[\left(1-\alpha^{*} e^{-j \Omega}\right)\left(\boldsymbol{I}-\eta \boldsymbol{R}_{\boldsymbol{S}}\right) \boldsymbol{\Sigma}\right]\right\},
\end{aligned}
$$

where $\eta=\sqrt{\frac{2}{\pi\left(\zeta+\sigma_{n}^{2}\right)}}$. Solving this equation for $\zeta$, we obtain the EMSE.

Finally, we achieve the following expressions for the EMSE of the second order methods.

$\zeta^{\mathrm{LCRLS}}=v_{i} \zeta_{e_{m}}+\left(1-v_{i}\right) \zeta_{e_{m}\left|e_{m}\right|^{2}}$,

and

$\zeta^{\mathrm{LCRLA}}=v_{i} \zeta_{\operatorname{csgn}\left(e_{m}\right)}+\left(1-v_{i}\right) \zeta_{e_{m}}$.

\subsection{Verification of the analytical results}

We use a random 10-tap channel to transmit 10000 bits generated by a Turyn sequence [45] (without pule shaping), and we consider that the channel follows the model of (16), where $\sigma_{q}=10^{-4}$, $\Omega=10^{-5}$ and $\alpha=0.9$. The Gaussian part of the noise has a variance of $10^{-6}$, while that of the impulsive part is $10^{-2}$. We average the results over 30 iterations. According to the results in Figs. 3 and 4 , we see that there is a good match between the theoretical and simulation results.

\section{Simulation results}

\subsection{Setup}

In this section, we examine the performance of our algorithm under a highly realistic underwater acoustic channel equalization scenario through a highly accurate modeling of the underwater 


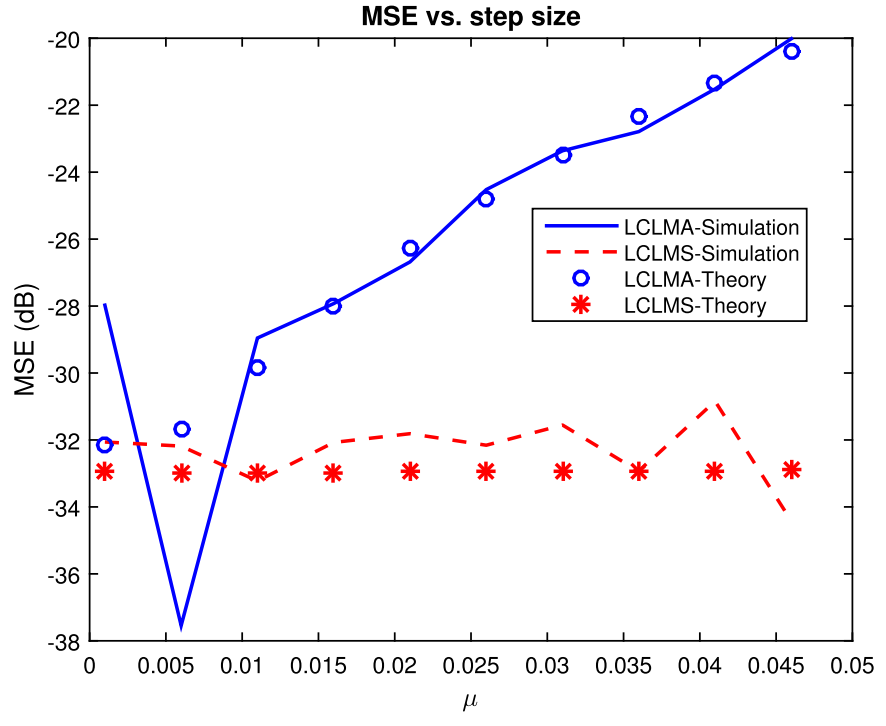

Fig. 3. The comparison between the simulation results and theoretical results for the MSE of our first order methods.

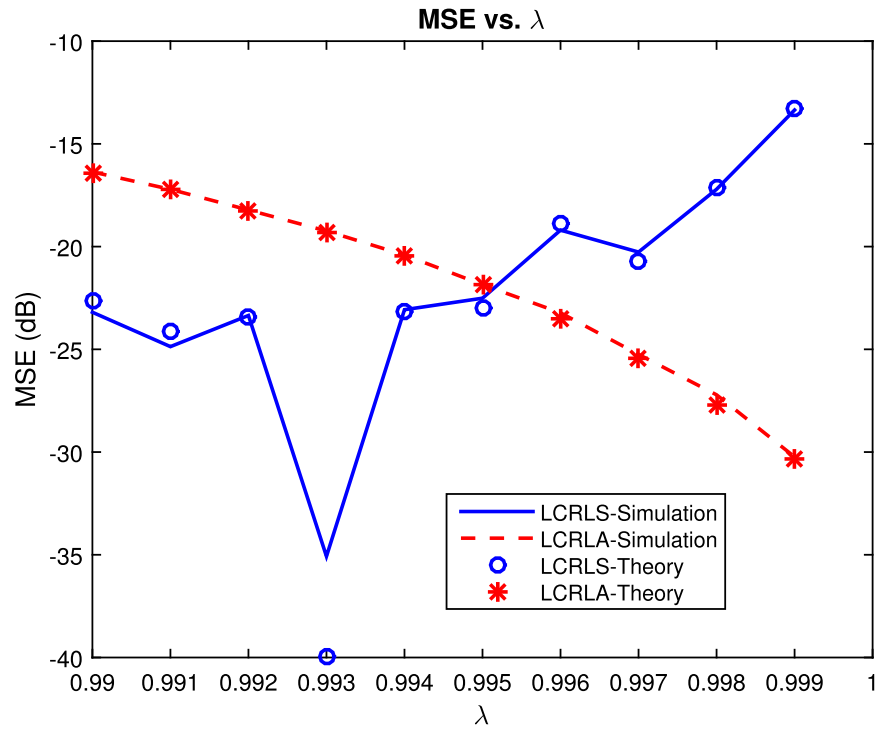

Fig. 4. The comparison between the simulation results and theoretical results for the MSE of our second order methods.

channels introduced in [46]. We add impulsive noise to the channel output to compare the robustness of the algorithms against impulsive noise. The simulation configurations and the parameters used for simulating the channel are presented in the Table 1. We send 60000 bits generated by repeating a Turyn sequence [45] (with a length of 28 bits), over the simulated UWA channel shown in Fig. 5. In addition, the system setup is the same as the one described at Section 2.2. Also, we calculate the SNR after down converting and matched filtering (i.e., from the baseband signal). The step sizes are set to $\mu=0.01$ for all algorithms. In all of the algorithms we use a 10-tap NLMS equalizer, with the causal and anti-causal parts each of length 5 . The length of the channel estimator is set to 140 , since according to Fig. $5, \tau_{\max } \approx 35 \mathrm{~ms}$ and $T_{s}=1 / 4 \mathrm{~ms}$ (because the symbol rate is $4 \mathrm{kHz})$.

We use a raised cosine pulse shaped signal with a roll-off factor of 0.25 and transmit the data at a rate of $4 \mathrm{k} \frac{\text { symbol }}{\text { second }}$, i.e., each sym-
Table 1

The simulated channel configurations.

\begin{tabular}{ll}
\hline Parameters & Values \\
\hline Transmitter (Tx) depth & $80 \mathrm{~m}$ \\
Receiver (Rx) depth & $50 \mathrm{~m}$ \\
Distance between Tx and Rx & $1 \mathrm{~km}$ \\
Carrier frequency $\left(f_{c}\right)$ & $15 \mathrm{kHz}$ \\
Signal bandwidth & $5 \mathrm{kHz}$ \\
Sample rate & $16 \mathrm{kHz}$ \\
Frequency resolution $(d f)$ & $16 \mathrm{~Hz}$ \\
Time resolution $(d t)$ & $1 / 16 \mathrm{~ms}$ \\
Symbol rate & $4 \mathrm{kHz}$ \\
Maximum multipath delay $\left(\tau_{\max }\right)$ & $35 \mathrm{~ms}$ \\
Coherence time of the small scale variants $\left(T_{S S}\right)$ & $0.4 \mathrm{~s}$ \\
Total duration of simulated channel $\left(T_{\text {tot }}\right)$ & $16 \mathrm{~s}$ \\
\hline
\end{tabular}

bol has a duration of $0.25 \mathrm{~ms}$. Since we use the BPSK modulation, the signal bandwidth is $B W=(1+\beta) R=(1+0.25) \times 4 \mathrm{kHz}=$ $5 \mathrm{kHz}$, where $\beta$ and $R$ are roll-off factor and the transmitting rate, respectively. We use 4 samples to represent each symbol, hence, the time resolution of the signal (the time distance between two signal samples) is $T_{s}=1 / 16 \mathrm{~ms}$.

In order to simulate the channel, we should use a time resolution $d t=T_{s}=1 / 16 \mathrm{~ms}$, which corresponds to using a sample rate of $16 \mathrm{kHz}$. Moreover, in practice, the maximum delay of an underwater channel is usually less than $50 \mathrm{~ms}$ [46], hence, we will observe $T_{o b s}=62.5 \mathrm{~ms}$ of the channel. One may note that the choice of $T_{o b s}$ implies the frequency resolution we use for simulating the channel, i.e., $d f=1 / T_{o b s}=16 \mathrm{~Hz}$. Note that since the maximum delay of the channel is around $35 \mathrm{~ms}$, the effective channel length is almost $35 / 0.25=140$ symbol durations. Also, we use a coherence time of $T_{S S}=400 \mathrm{~ms}$, since according to [5], for a general purpose design one should consider a coherence time of several hundred milliseconds. Furthermore, the sound speed in the water for this experiment is $c=1500 \mathrm{~m} / \mathrm{s}$ [47] and the spreading factor is set to $k=1.7$ [46].

The results are averaged over 30 repetitions, and show the superior performance of our robust algorithm over other methods. We compare the bit error rate (BER) as well as the normalized time mean squared errors (MSE) of different algorithms to show the efficacy of our methods. In particular, to precisely evaluate the tracking performance of the algorithms, we compute the MSE exactly after the causal ISI removal block, i.e., the MSE at time step $n$ is defined as MSE $\triangleq \frac{1}{n} \sum_{m=1}^{n}\left(s_{m}-\tilde{r}_{m}\right)^{2}$.

\subsection{Results and discussion}

We perform two experiments to evaluate the performance of the algorithms in (1) different SNR values (2) and different impulse probabilities. In the first experiment, in order to indicate the BER vs. SNR performance of our algorithms, i.e, LCLMA, LCLMS, LCRLS and LCRLA, we use a mixture of Gaussian and a $5 \%$ impulsive noise model, i.e., $v_{i}=0.05$, with the variance (power) of the impulsive noise being set to $10^{4}$ times that of the Gaussian noise. In these experiments, we compare the BER and MSE of our methods with those of the conventional DFE and the state-of-the-art algorithms in the literature including: reweighted zero-attracting least mean p-power (RZALMP) [35], improved least sum of exponentials (ILSE) [34], $l_{0}$-RLS [32] (indicated as LZRLS in the figures), as well as the conventional SA, RLS and LMS algorithms. We emphasize that all of these algorithms are designed to combat the impulsive noise through minimization of different cost functions summarized in Table 2. For the sake of clarity, we demonstrate the results of each experiment in two different plots, one for the first order algorithms, i.e., LCLMA, LCLMS, LMS, SA, ILSE, RZALMP and another for the second order algorithms, i.e., LCRLA, LCRLS, RLS 


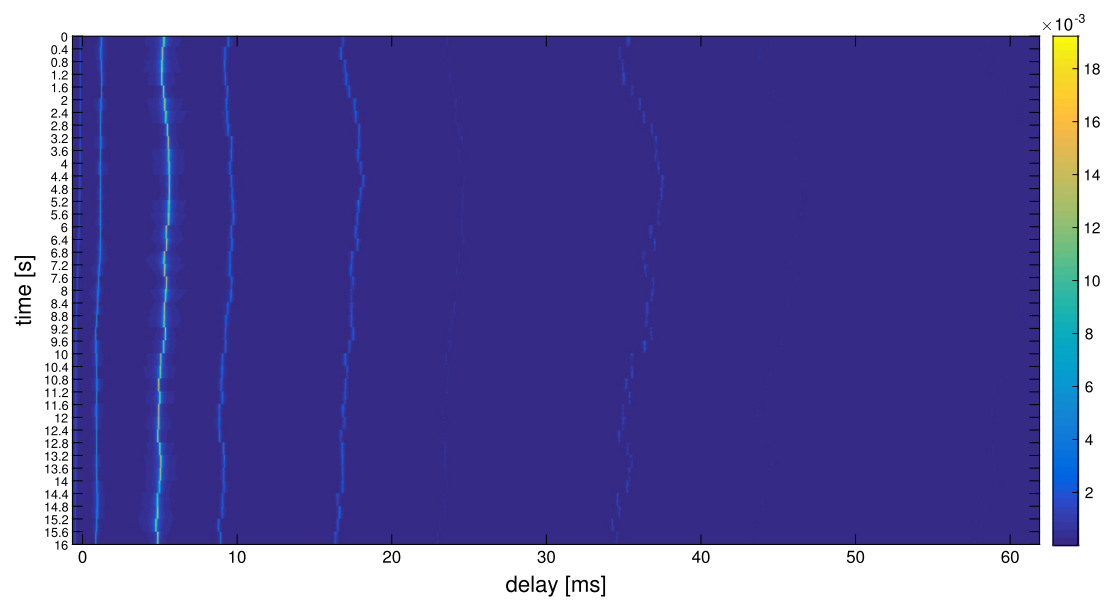

Fig. 5. Time evolution of the magnitude baseband impulse response of the generated channel [46].

Table 2

Cost functions of the competing algorithms.

\begin{tabular}{ll}
\hline Algorithm & Cost function \\
\hline SA & $\left|e_{m}\right|$ \\
LMS & $\left|e_{m}\right|^{2}$ \\
LCLMS & $\left|e_{m}\right|^{2}-\frac{1}{a} \ln \left(1+a\left|e_{m}\right|^{2}\right)$ \\
LCLMA & $\left|e_{m}\right|-\frac{1}{a} \ln \left(1+a\left|e_{m}\right|\right)$ \\
ILSE & $\frac{1}{\lambda} \cosh \left(\lambda e_{m}\right)$ \\
RZALMP & $\left|e_{m}\right|^{p}+\lambda \sum_{i=1}^{L} \log \left(1+\frac{\left|\hat{h}_{i}\right|}{\delta}\right)$ \\
RLS & $\sum_{i=1}^{m} \lambda^{m-i}\left|e_{i}\right|^{2}$ \\
LCRLS & $\sum_{i=1}^{m} \lambda^{m-i}\left|e_{i}\right|^{2}-\frac{1}{a} \ln \left(1+a \sum_{i=1}^{m} \lambda^{m-i}\left|e_{i}\right|^{2}\right)$ \\
LCRLA & $\sum_{i=1}^{m} \lambda^{m-i}\left|e_{i}\right|-\frac{1}{a} \ln \left(1+a \sum_{i=1}^{m} \lambda^{m-i}\left|e_{i}\right|\right)$ \\
$l_{0}$-RLS & $\sum_{i=0}^{m} \lambda^{m-i}\left|e_{i}\right|^{2}+\zeta\left\|\hat{\mathbf{h}}_{n}\right\|_{0}$, where $\left\|\hat{\mathbf{h}}_{n}\right\|_{0} \approx \sum_{k=0}^{K-1}\left(1-\exp \left(-\eta\left|\hat{h}_{k}\right|\right)\right)$ \\
\hline
\end{tabular}

Table 3

Computational complexity comparison of different algorithms. Number of each operation (on real numbers) needed by different algorithms per one sample processing is provided in the table. We have assumed that the exponentiating to a non-integer number needs $M$ multiplication and $N$ additions.

\begin{tabular}{lllll}
\hline Algorithm & $\times$ & + & sign \\
\hline SA & $6 L$ & $4 L$ & & 2 \\
LMS & $8 L+2$ & $8 L$ & & \\
LCLMS & $2 L+6$ & $2 L+1$ & 1 & \\
LCLMA & $6 L+3$ & $4 L+1$ & 1 & 2 \\
ILSE & $6 L+2 M+2$ & $4 L+2 N+1$ & 1 & \\
RZALMP & $12 L+M$ & $7 L+N$ & $2 L$ & $2 L+2$ \\
RLS & $14 L^{2}+10 L+1$ & $12 L^{2}+4 L-1$ & $2 L+1$ & \\
LCRLS & $14 L^{2}+10 L+4$ & $12 L^{2}+4 L$ & $2 L+2$ & \\
LCRLA & $14 L^{2}+10 L+2$ & $12 L^{2}+4 L$ & $2 L+2$ & \\
$l_{0}$-RLS & $30 L^{2}+11 L+L M+4$ & $29 L^{2}-4 L+L N+2$ & $2 L+3$ & $2 L$ \\
\hline
\end{tabular}

and $l_{0}$-RLS. We have also provided the complexity comparisons of different methods in Table 3.

As shown in the Figs. 6 and 7, our algorithms outperform all other algorithms in all SNR values. Also, observe that the conventional DFE cannot perform well in lower SNRs, while in high SNRs, it delivers a comparable performance to our methods. Moreover, the Figs. 8 and 9 depict the MSE results for the first order and second order algorithms over time at SNR $=20 \mathrm{~dB}$, respectively (one can observe the MSE comparison at first time steps in Figs. 10 and 11). Again, the MSE results in Figs. 8 and 9, show how our algorithms can successfully track the channel variations in this highly

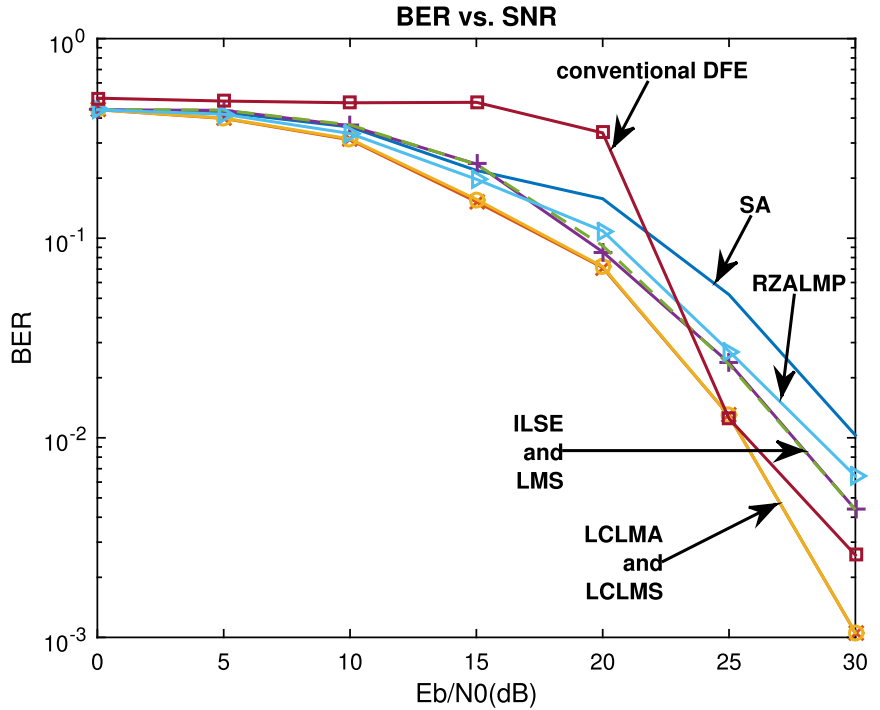

Fig. 6. BER vs SNR of the first order algorithms, in a $5 \%$ impulsive noise environment. This figure shows the superior performance of LCLMS and LCLMA algorithms.

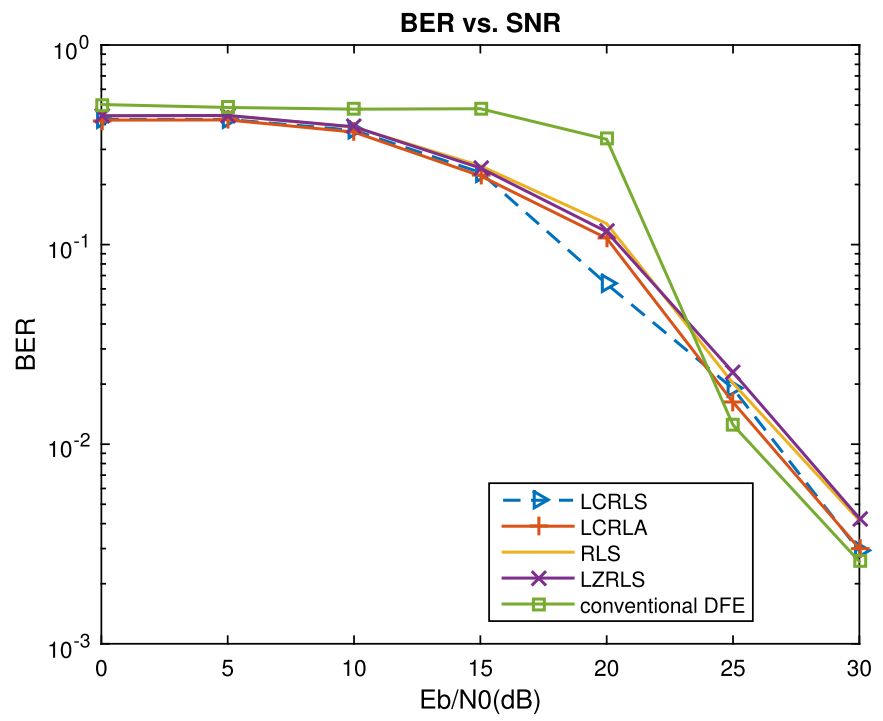

Fig. 7. BER vs SNR of the second order algorithms, in a $5 \%$ impulsive noise environment. This figure shows the superior performance of LCRLS algorithm. 


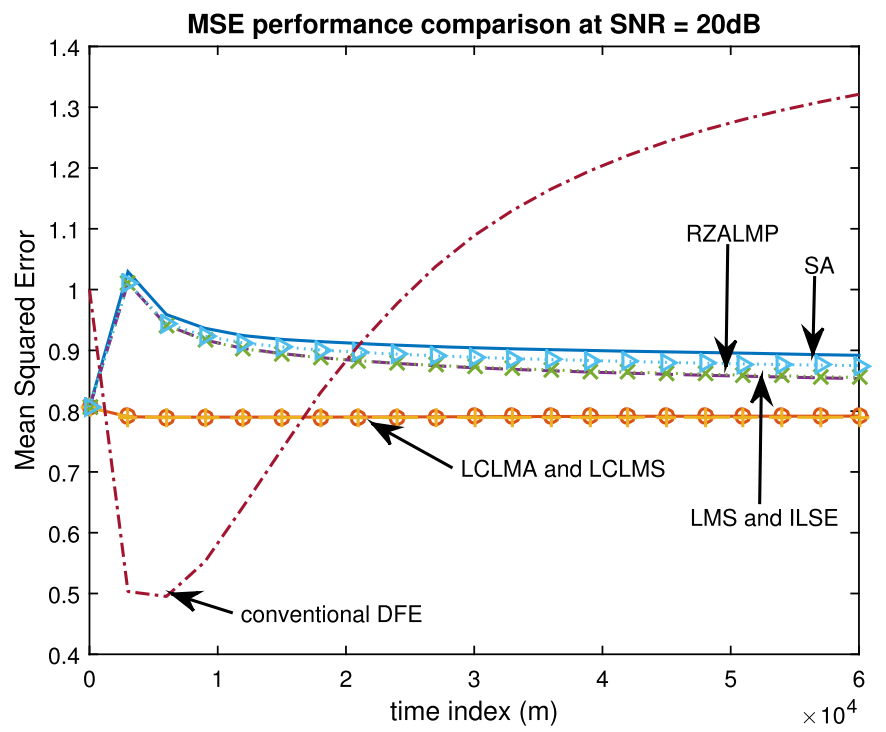

Fig. 8. MSE of the first order algorithms, in $5 \%$ impulsive noise environment. This figure shows the superior convergence performance of the LCLMS and LCLMA methods at $\mathrm{SNR}=20 \mathrm{~dB}$

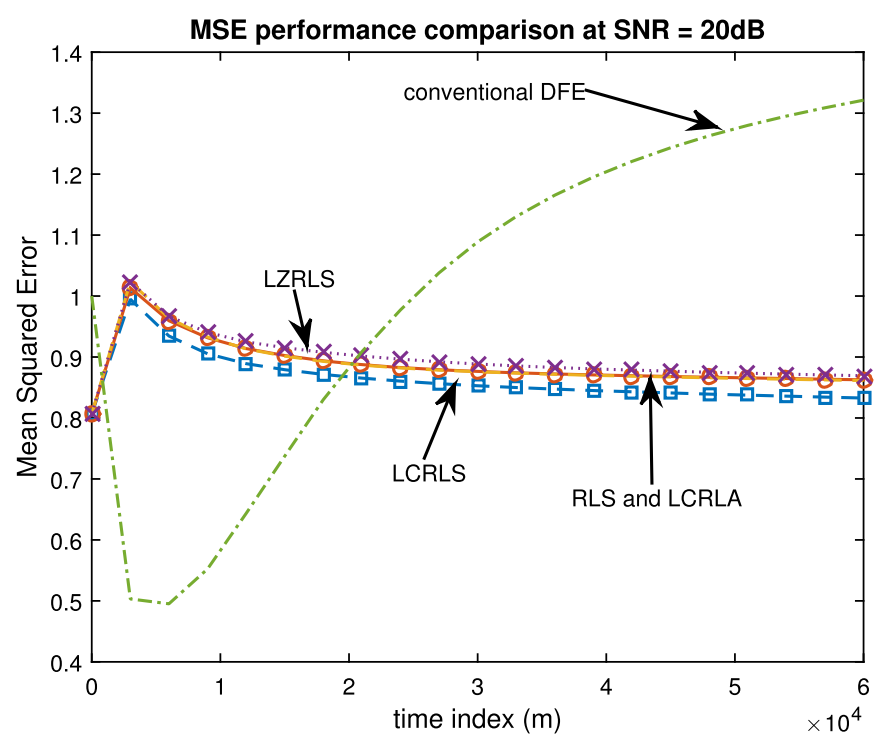

Fig. 9. MSE of the second order algorithms, in $5 \%$ impulsive noise environment This figure shows the superior convergence performance of the LCRLS method at $\mathrm{SNR}=20 \mathrm{~dB}$.

non-stationary and impulsive noise environment, yielding a superior performance related to the other competitors.

We also investigate the effect of the impulse probability, $v_{i}$, on the performance of the proposed algorithms. To this end, we set the SNR to $20 \mathrm{~dB}$ and obtain the BER at different impulse probabilities, as shown in Figs. 12 and 13. Based on these simulations, when the impulse probability increases, our algorithms significantly outperform other methods. This superior performance makes our algorithms suitable candidates for the highly impulsive noise real life channels.

\section{Conclusion}

In this paper, we presented a novel family of linear channel estimation algorithms based on the logarithmic cost functions. Specifically, we introduce two first order methods, i.e., LCLMA

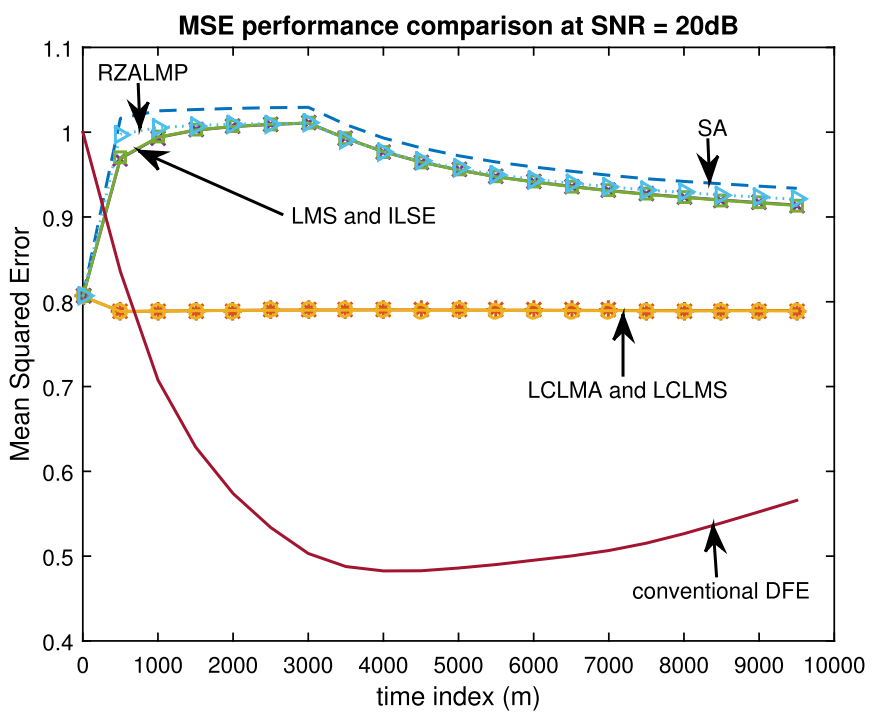

Fig. 10. MSE comparison for different first order methods.

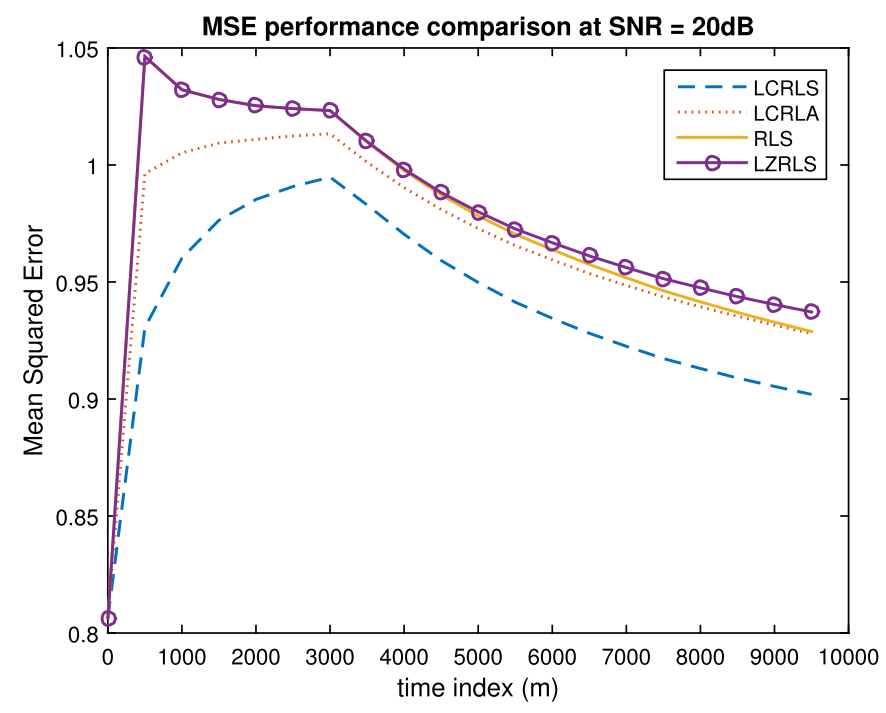

Fig. 11. MSE comparison for different second order methods.

and LCLMS, as well as two second order methods, i.e., LCRLA and LCRLS, as robust adaptive estimators for underwater acoustic channels. We implement these algorithms in a decision feedback equalization framework and remove the ISI in two consecutive stages, i.e., a channel estimation based equalizer followed by a blind equalizer. Our methods achieve a comparable convergence rate to the algorithms seeking to minimize higher order norms of the error, while maintaining the same stability of the lower order norm methods, with similar computational complexity to the conventional methods. We provide the tracking and steadystate performance analysis of the proposed algorithms both in the impulse-free and impulsive noise environments, in the presence of frequency and phase offsets, which are among the common impairments in underwater acoustic communications. Finally, we show the enhanced performance of the new algorithms in a highly realistic UWA communication scenario. 


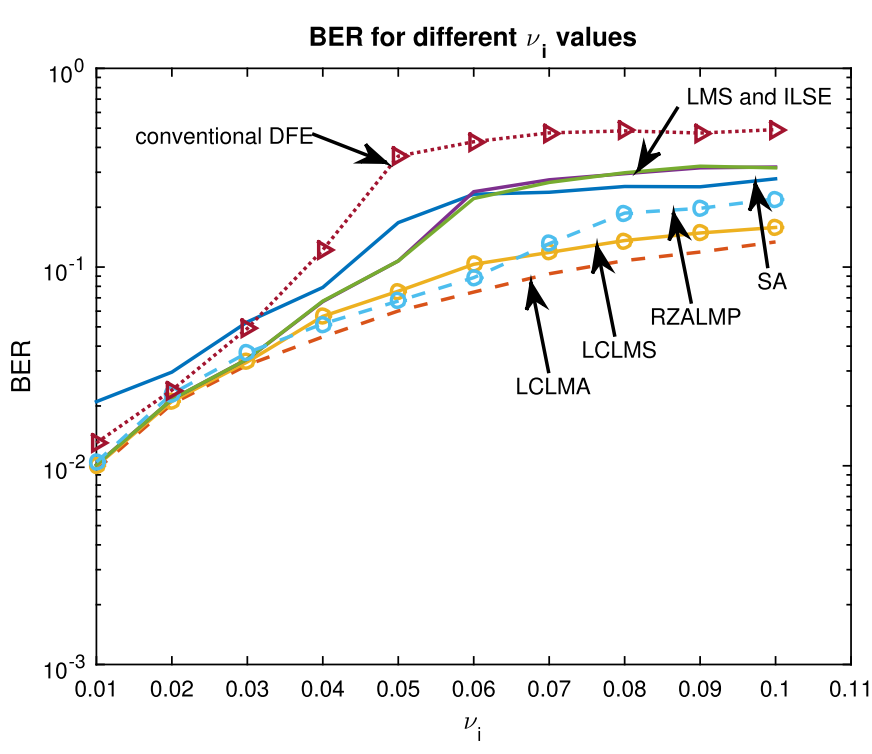

Fig. 12. BER at different impulse probabilities for the first order algorithms. The experiments are done at $\mathrm{SNR}=20 \mathrm{~dB}$.

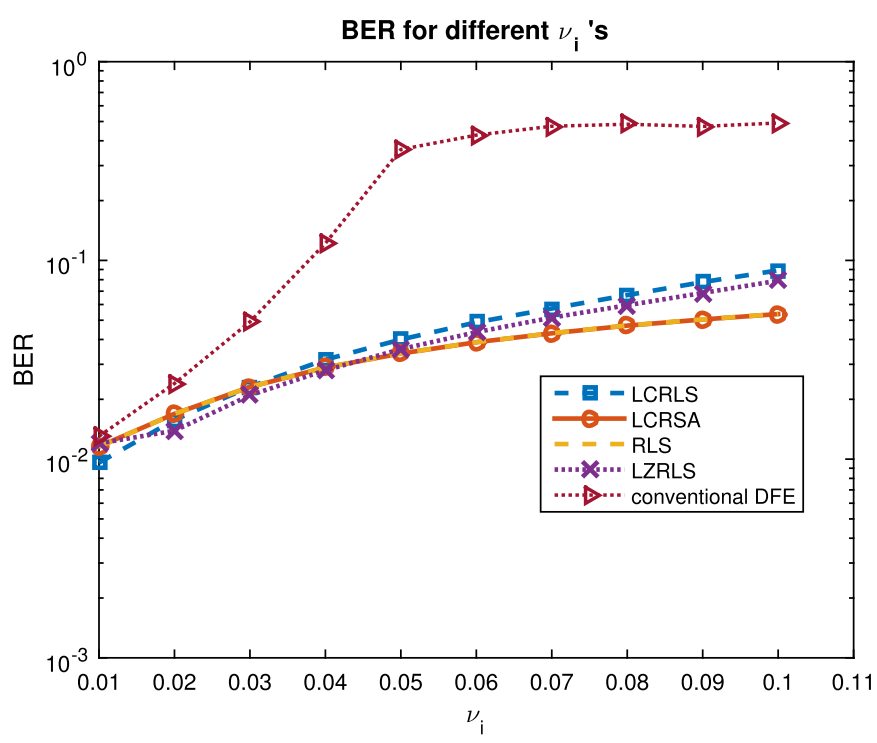

Fig. 13. BER at different impulse probabilities for the second order algorithms. The experiments are done at $\mathrm{SNR}=20 \mathrm{~dB}$.

\section{Acknowledgments}

This work is in part supported by Turkish Academy of Sciences, Outstanding Researcher Programme and TUBITAK, Contract No.: $112 \mathrm{E} 161$.

\section{Appendix A. Supplementary material}

Supplementary material related to this article can be found online at http://dx.doi.org/10.1016/j.dsp.2017.05.006.

\section{References}

[1] M.K. Banavar, J.J. Zhang, B. Chakraborty, H. Kwon, Y. Li, H. Jiang, A. Spanias, C. Tepedelenlioglu, C. Chakrabarti, A. Papandreou-Suppappola, An overview of recent advances on distributed and agile sensing algorithms and implementation, Digit. Signal Process. 39 (2015) 1-14, http://dx.doi.org/10.1016/ j.dsp.2015.01.001.

[2] A. Gunes, M.B. Guldogan, Joint underwater target detection and tracking with the Bernoulli filter using an acoustic vector sensor, Digit. Signal Process. 48 (2016) 246-258, http://dx.doi.org/10.1016/j.dsp.2015.09.020.
[3] A.C. Singer, J.K. Nelson, S.S. Kozat, Signal processing for underwater acoustic communications, IEEE Commun. Mag. 47 (1) (2009) 90-96.

[4] D.B. Kilfoyle, A.B. Baggeroer, The state of the art in underwater acoustic telemetry, IEEE J. Ocean. Eng. 25 (1) (2000) 4-27, http://dx.doi.org/10.1109/48.820733.

[5] M. Stojanovic, J. Preisig, Underwater acoustic communication channels: propagation models and statistical characterization, IEEE Commun. Mag. 47 (1) (2009) 84-89.

[6] D. Peng, Y. Xiang, H. Trinh, Z. Man, Adaptive blind equalization of time-varying SIMO systems driven by QPSK inputs, Digit. Signal Process. 23 (1) (2013) 268-274, http://dx.doi.org/10.1016/j.dsp.2012.07.014.

[7] J.G. Proakis, Digital Communications, McGraw-Hill, 1995

[8] A. Song, M. Badiey, Generalized equalization for underwater acoustic communications, in: Proceedings of OCEANS 2005 MTS/IEEE, 2005, pp. 1522-1527.

[9] Y.R. Zheng, V.H. Nascimento, Two variable step-size adaptive algorithms for non-Gaussian interference environment using fractionally lower-order moment minimization, Digit. Signal Process. 23 (3) (2013) 831-844.

[10] X. Xu, S. Zhou, H. Sun, A.K. Morozov, Y. Zhang, Impulsive noise suppression in per-survivor processing based DSSS systems, in: Oceans, St. John's 2014, 2014, pp. $1-5$.

[11] S. Banerjee, M. Agrawal, Underwater acoustic communication in the presence of heavy-tailed impulsive noise with bi-parameter Cauchy-Gaussian mixture model, in: Ocean Electronics, SYMPOL 2013, 2013, pp. 1-7.

[12] S. Banerjee, M. Agrawal, Underwater acoustic noise with generalized Gaussian statistics: effects on error performance, in: OCEANS, Bergen, 2013 MTS/IEEE, 2013, pp. $1-8$.

[13] S. Banerjee, M. Agrawal, On the performance of underwater communication system in noise with Gaussian mixture statistics, in: 2014 Twentieth National Conference on Communications, NCC, 2014, pp. 1-6.

[14] T.Y. Min, M. Chitre, Localization of impulsive sources in the ocean using the method of images, in: Oceans, St. John's, 2014, 2014, pp. 1-6.

[15] D. Zha, T. Qiu, Underwater sources location in non-Gaussian impulsive noise environments, Digit. Signal Process. 16 (2) (2006) 149-163, http://dx.doi.org/ 10.1016/j.dsp.2005.04.008.

[16] J. He, Z. Liu, Underwater acoustic azimuth and elevation angle estimation using spatial invariance of two identically oriented vector hydrophones at unknown locations in impulsive noise, Digit. Signal Process. 19 (3) (2009) 452-462, http://dx.doi.org/10.1016/j.dsp.2007.10.012.

[17] J. Zhang, Y. Pang, Pipelined robust m-estimate adaptive second-order Volterra filter against impulsive noise, Digit. Signal Process. 26 (2014) 71-80.

[18] J.A. Hildebrand, Anthropogenic and natural sources of ambient noise in the ocean, Inter-Research, Mar. Ecol. Prog. Ser. 395 (2009) 5-20, http://dx.doi.org/ 10.3354/meps08353.

[19] H.-Y. Cheng, A.-Y.A. Wu, Unified low-complexity decision feedback equalizer with adjustable double radius constraint, Digit. Signal Process. 51 (C) (2016) 82-91, http://dx.doi.org/10.1016/j.dsp.2016.01.012.

[20] Z. Madadi, G.V. Anand, A.B. Premkumar, Three-dimensional localization of multiple acoustic sources in shallow ocean with non-Gaussian noise, Digit. Signal Process. 32 (2014) 85-99, http://dx.doi.org/10.1016/j.dsp.2014.05.002.

[21] M.U. Otaru, A. Zerguine, L. Cheded, Channel equalization using simplified least mean-fourth algorithm, Digit. Signal Process. 21 (3) (2011) 447-465, http:// dx.doi.org/10.1016/j.dsp.2010.11.005.

[22] A. Zerguine, Convergence and steady-state analysis of the normalized least mean fourth algorithm, Digit. Signal Process. 17 (1) (2007) 17-31, http:// dx.doi.org/10.1016/j.dsp.2006.01.005.

[23] M.O. Sayin, N.D. Vanli, S.S. Kozat, A novel family of adaptive filtering algorithms based on the logarithmic cost, IEEE Trans. Signal Process. 62 (17) (2014) 4411-4424, http://dx.doi.org/10.1109/TSP.2014.2333559.

[24] S. Haykin, Adaptive Filter Theory, 4th edition, Prentice Hall Inf. Syst. Sci. Ser., Prentice Hall, Upper Saddle River, N.J., 2002.

[25] A.H. Sayed, Fundamentals of Adaptive Filtering, John Wiley \& Sons, NJ, 2003.

[26] J.A. Chambers, O. Tanrikulu, A.G. Constantinides, Least mean mixed-norm adaptive filtering, Electron. Lett. 30 (19) (1994) 1574-1575, http://dx.doi.org/ 10.1049/el:19941060.

[27] J. Chambers, A. Avlonitis, A robust mixed-norm adaptive filter algorithm, IEEE Signal Process. Lett. 4 (2) (1997) 46-48, http://dx.doi.org/10.1109/97.554469.

[28] J. Arenas-Garcia, V. Gomez-Verdejo, A.R. Figueiras-Vidal, New algorithms for improved adaptive convex combination of LMS transversal filters, IEEE Trans. Instrum. Meas. 54 (6) (2005) 2239-2249, http://dx.doi.org/10.1109/ TIM.2005.858823.

[29] J. Arenas-Garcia, A.R. Figueiras-Vidal, A.H. Sayed, Mean-square performance of a convex combination of two adaptive filters, IEEE Trans. Signal Process. 54 (3) (2006) 1078-1090, http://dx.doi.org/10.1109/TSP.2005.863126.

[30] M.T.M. Silva, V. Nascimento, Improving the tracking capability of adaptive filters via convex combination, IEEE Trans. Signal Process. 56 (7) (2008) 3137-3149, http://dx.doi.org/10.1109/TSP.2008.919105.

[31] S.S. Kozat, A.T. Erdogan, A.C. Singer, A.H. Sayed, Steady-state MSE performance analysis of mixture approaches to adaptive filtering, IEEE Trans. Signal Process. 58 (8) (2010) 4050-4063, http://dx.doi.org/10.1109/TSP.2010.2049650.

[32] K. Pelekanakis, M. Chitre, Adaptive sparse channel estimation under symmetric alpha-stable noise, IEEE Trans. Wirel. Commun. 13 (6) (2014) 3183-3195, http://dx.doi.org/10.1109/TWC.2014.042314.131432. 
[33] K. Pelekanakis, M. Chitre, Robust equalization of mobile underwater acoustic channels, IEEE J. Ocean. Eng. 40 (4) (2015) 775-784, http://dx.doi.org/ 10.1109/JOE.2015.2469895.

[34] S. Wang, Y. Zheng, S. Duan, L. Wang, C.K. Tse, A class of improved least sum of exponentials algorithms, Signal Process. 128 (2016) 340-349, http://dx.doi.org/10.1016/j.sigpro.2016.05.005, http:// www.sciencedirect.com/science/article/pii/S0165168416300573.

[35] W. Ma, B. Chen, H. Qu, J. Zhao, Sparse least mean p-power algorithms for channel estimation in the presence of impulsive noise, Signal Image Video Process. 10 (3) (2016) 503-510

[36] C. van den Bos, M.H.L. Ksuwenhoven, W.A. Serdijn, Effect of smooth nonlinear distortion on OFDM symbol error rate, IEEE Trans. Commun. 49 (9) (2001) 1510-1514, http://dx.doi.org/10.1109/26.950336.

[37] P. Banelli, Bayesian estimation of a Gaussian source in Middleton's class a impulsive noise, IEEE Signal Process. Lett. 20 (10) (2013) 956-959, http:// dx.doi.org/10.1109/LSP.2013.2274774.

[38] L. Rugini, P. Banelli, On the equivalence of maximum SNR and MMSE estimation: applications to additive non-Gaussian channels and quantized observations, IEEE Trans. Signal Process. 64 (23) (2016) 6190-6199, http://dx.doi.org/ 10.1109/TSP.2016.2607152.

[39] N.R. Yousef, A.H. Sayed, Ability of adaptive filters to track carrier offsets and channel nonstationarities, IEEE Trans. Signal Process. 50 (7) (2002) 1533-1544 http://dx.doi.org/10.1109/TSP.2002.1011194.

[40] F. Qu, L. Yang, Basis expansion model for underwater acoustic channels?, in: OCEANS 2008, 2008, pp. 1-7.

[41] C.P. Shah, C.C. Tsimenidis, B.S. Sharif, J.A. Neasham, Low-complexity iterative receiver structure for time-varying frequency-selective shallow underwater acoustic channels using BICM-ID: design and experimental results, IEEE J. Ocean. Eng. 36 (3) (2011) 406-421, http://dx.doi.org/10.1109/JOE.2011.2144670.

[42] X. Wang, H.V. Poor, Joint channel estimation and symbol detection in Rayleigh flat-fading channels with impulsive noise, IEEE Commun. Lett. 1 (1) (1997) 19-21, http://dx.doi.org/10.1109/4234.552144.

[43] S.-C. Chan, Y.-X. Zou, A recursive least M-estimate algorithm for robust adaptive filtering in impulsive noise: fast algorithm and convergence performance analysis, IEEE Trans. Signal Process. 52 (4) (2004) 975-991, http://dx.doi.org/ 10.1109/TSP.2004.823496.

[44] T.Y. Al-Naffouri, A.H. Sayed, Transient analysis of adaptive filters with error nonlinearities, IEEE Trans. Signal Process. 51 (3) (2003) 653-663, http:// dx.doi.org/10.1109/TSP.2002.808108.

[45] R.J. Turyn, Sequences with small correlation, in: Error Correcting Codes: Proceedings of a Symposium, 1968, pp. 195-228.

[46] P. Qarabaqi, M. Stojanovic, Statistical characterization and computationally efficient modeling of a class of underwater acoustic communication channels, IEEE J. Ocean. Eng. 38 (4) (2013) 701-717.

[47] P.C. Etter, Underwater Acoustic Modeling and Simulation, 4th edition, 2013.

Dariush Kari received the B.S. degree with high honor in two majors, Electrical Engineering and Computer Science, from Amirkabir University of Technology (Tehran Polytechnic), Tehran, Iran, July 2014. He is currently an M.S. student at the Department of Electrical and Electronics Engineering, Bilkent University, Ankara, Turkey. His current research interests include learning theory, online learning, big data analytics, optimization, statistical signal processing, and adaptive filtering.
Iman Marivani received the B.S. degree with high honor in Electrical Engineering, from Amirkabir University of Technology (Tehran Polytechnic), Tehran, Iran, April 2015. He is currently an M.S. student at the Department of Electrical and Electronics Engineering, Bilkent University Ankara, Turkey. His current research interests include anomaly detection, recommender systems, online learning, and big data analytics.

Farhan Khan was born in Swabi, Pakistan, in 1983. He received the B.Sc. degree with honors in Electrical Engineering from University of Engineering and Technology, Peshawar, Pakistan, in 2007, and the M.Sc. degree in RF Communication Systems from University of Southampton, UK, in 2009. After graduation, he joined the Department of Electrical Engineering at the COMSATS Institute of Information Technology, Pakistan. He is currently working towards the Ph.D. degree in the Department of Electrical and Electronics Engineering at Bilkent University, Ankara, Turkey. His research interests include adaptive signal processing, big data, machine learning, and neural networks.

Muhammed Omer Sayin received the B.S. and M.S. degrees in electrical and electronics engineering from Bilkent University, Ankara, Turkey in 2013 and 2015, respectively. He is currently pursuing the Ph.D. degree in electrical and computer engineering with the University of Illinois at Urbana-Champaign, Champaign, IL, USA. His current research interests include distributed networks, optimal control, and dynamic games.

Suleyman Serdar Kozat (A'10-M'11-SM'11) received the B.S. degree with full scholarship and high honors from Bilkent University, Turkey. He received the M.S. and Ph.D. degrees in electrical and computer engineering from University of Illinois at Urbana-Champaign, Urbana, IL. Dr. Kozat is a graduate of Ankara Fen Lisesi. After graduation, Dr. Kozat joined IBM Research, T.J. Watson Research Lab, Yorktown, New York, US as a Research Staff Member in the Pervasive Speech Technologies Group. While doing his Ph.D., he was also working as a Research Associate at Microsoft Research, Redmond, Washington, US in the Cryptography and Anti-Piracy Group. He holds several patent inventions due to his research accomplishments at IBM Research and Microsoft Research. Dr. Kozat is currently an Associate Professor at the Electrical And Electronics Engineering Department of Bilkent University. Dr. Kozat is the President of the IEEE Signal Processing Society, Turkey Chapter. He has been elected to the IEEE Signal Processing Theory and Methods Technical Committee and IEEE Machine Learning for Signal Processing Technical Committee, 2013. He has been awarded IBM Faculty Award by IBM Research in 2011, Outstanding Faculty Award by Koc University in 2011 (granted the first time in 16 years), Outstanding Young Researcher Award by the Turkish National Academy of Sciences in 2010, ODTU Prof. Dr. Mustafa N. Parlar Research Encouragement Award in 2011, Outstanding Faculty Award by Bilim Kahramanlari, 2013 and holds Career Award by the Scientific Research Council of Turkey, 2009. 